\title{
Administration of a CXC Chemokine Receptor 2 (CXCR2) Antagonist, SCH527123, Together with Oseltamivir Suppresses NETosis and Protects Mice from Lethal Influenza and Piglets from Swine-Influenza Infection
}

\author{
Harshini K. Ashar, ${ }^{*}$ Sivasami Pulavendran, ${ }^{*}$ Jennifer M. Rudd, * Prasanthi Maram, ${ }^{*}$ Mallika Achanta, Vincent T.K. Chow, \\ Jerry R. Malayer, ${ }^{*}$ Timothy A. Snider, ${ }^{*}$ and Narasaraju Teluguakula*
}

From the Center for Veterinary Health Sciences, * Oklahoma State University, Stillwater, Oklahoma; and the National University Health System Infectious Diseases Translational Research Program, ${ }^{\dagger}$ Department of Microbiology and Immunology, School of Medicine, National University of Singapore, Singapore

\author{
Accepted for publication \\ December 22, 2020. \\ Address correspondence to \\ Narasaraju Teluguakula, \\ Ph.D., College of Veterinary \\ Medicine, Stillwater, \\ OK 74078. E-mail: narasa@ \\ okstate.edu.
}

\begin{abstract}
Excessive neutrophil influx, their released neutrophil extracellular traps (NETs), and extracellular histones are associated with disease severity in influenza-infected patients. Neutrophil chemokine receptor CXC chemokine receptor 2 (CXCR2) is a critical target for suppressing neutrophilic inflammation. Herein, temporal dynamics of neutrophil activity and NETosis were investigated to determine the optimal timing of treatment with the CXCR2 antagonist, SCH527123 (2-hydroxy-N,N-dimethyl-3-[2-([(R)-1-(5-methylfuran-2-yl)-propyl]amino)-3,4-dioxo-cyclobut-1-enylamino]-benzamide), and its efficacy together with antiviral agent, oseltamivir, was tested in murine and piglet influenza-pneumonia models. SCH527123 plus oseltamivir markedly improved survival of mice infected with lethal influenza, and diminished lung pathology in swine-influenza-infected piglets. Mechanistically, addition of SCH527123 in the combination treatment attenuated neutrophil influx, NETosis, in both mice and piglets. Furthermore, neutrophils isolated from influenza-infected mice showed greater susceptibility to NETotic death when stimulated with a CXCR2 ligand, IL-8. In addition, CXCR2 stimulation induced nuclear translocation of neutrophil elastase, and enhanced citrullination of histones that triggers chromatin decondensation during NET formation. Studies on temporal dynamics of neutrophils and NETs during influenza thus provide important insights into the optimal timing of CXCR2 antagonist treatment for attenuating neutrophil-mediated lung pathology. These findings reveal that pharmacologic treatment with CXCR2 antagonist together with an antiviral agent could significantly ameliorate morbidity and mortality in virulent and sublethal influenza infections. (Am J Pathol 2021, 191: 669-685; https://doi.org/10.1016/ j.ajpath.2020.12.013)
\end{abstract}

Influenza virus infections during pandemic outbreaks and yearly seasonal epidemics cause significant morbidity and mortality rates globally. ${ }^{1}$ Seasonal influenza-associated deaths have increased in recent years, with an estimated of $>600,000$ fatalities annually. ${ }^{2}$ A significant proportion of hospitalized patients with influenza develop complications of acute respiratory distress syndrome, characterized by widespread alveolar-capillary injury, inflammation, edema, and parenchymal hemorrhage. ${ }^{3-8}$ These pathologic manifestations are driven by virus-inflicted cytopathic effects as well as exaggerated host immune responses. ${ }^{9-11}$
Vaccination is the logical choice for controlling the virus. However, because of unrelenting emergence of new strains and their mutative ability, vaccination presents a major challenge during influenza outbreaks. ${ }^{12,13}$ In such cases, treatment primarily depends on antiviral therapy.

\footnotetext{
Supported by the National Institute of General Medical Sciences of the NIH under award number P20GM103648 (N.T.); an Oklahoma Center for the Advancement of Science and Technology grant (N.T.); and a Center for Veterinary Health Sciences, Oklahoma State University grant (N.T.).

H.K.A. and N.T. contributed equally to this work as first authors.

Disclosures: None declared.
} 
Administration of antiviral drugs may not always be effective, as considerable lung pathology is mediated by exaggerated host-immune responses in addition to virus-inflicted cytotoxicity. ${ }^{14-17}$

Previously, we established that massive neutrophil influx, their induced neutrophil extracellular traps (NETs), and extracellular histones (ECHs) aggravate pulmonary pathology in severe influenza. ${ }^{18-23}$ Aberrant neutrophil activity and accumulation of NETs are also documented in patients with severe influenza. ${ }^{24,25}$ Neutrophils are recruited to the site of injury/infection via chemokine signaling, mediated through chemokine receptors. Among various chemokine receptors, CXC chemokine receptor 2 (CXCR2) plays a critical role in modulating neutrophil functionality during influenza. ${ }^{26}$ Numerous clinical studies have also tested CXCR2 antagonists for their efficacy in reducing inflammation and organ injuries in acute and chronic diseases. ${ }^{27-31}$ Recently, human phase 2 trials evaluated the safety and efficacy of a CXCR2 antagonist, danirixin, alone or in combination with oseltamivir in influenza-infected patients. ${ }^{27,28}$ Although administration of danirixin was found to be safe and well-tolerated, no differences in the clinical scores were observed between patients given oseltamivir alone and those given danirixin plus oseltamivir. ${ }^{27}$ Furthermore, there was inconsistency in neutrophil numbers among different treatment groups. This inconsistency may be attributed to the absence of rational determination of the optimal timing and dosing of danirixin, to achieve the fine balance of suppressing excessive neutrophil influx, without compromising the beneficial host immunity by neutrophils. Moreover, the underlying mechanistic roles of targeting CXCR2 and its pathogenic association with influenza pneumonia have not been established.

NETs are large extracellular web-like chromatin strands that were initially proposed to have a defense mechanism against invading pathogens. ${ }^{32}$ However, excessive release of NETs aggravates tissue injury and death, as reported in several disease conditions. ${ }^{33-36}$ NETosis is regulated by various granule and nuclear proteins. ${ }^{37}$ Myeloperoxidase (MPO) and neutrophil elastase (NEs) are released from azurophilic granules, anchor chromatin scaffolds in NETs, and mediate histone degradation during NETosis. ${ }^{38}$ We reported earlier that blocking MPO decreases NETs, but signaling mechanisms in influenza-induced NETosis remain unclear. ${ }^{17,18}$ Herein, we evaluated the therapeutic efficacy of a CXCR2 antagonist, SCH527123 (2-hydroxy-N,Ndimethyl-3-[2-([(R)-1-(5-methyl-furan-2-yl)-propyl]amino)3,4-dioxo-cyclobut-1-enylamino]-benzamide) alone or in combination with antiviral agent, oseltamivir (which inhibits viral neuraminidase and prevents progeny virus release from infected cells), in models of lethal influenza-infected mice and sublethal swine influenza-infected piglets. SCH527123 plus oseltamivir significantly improved survival in lethal influenza-challenged mice, and attenuated lung pathology in swine influenza-infected piglets. Thus, SCH5277123 plus oseltamivir represents a promising combination treatment against influenza pneumonia.

\section{Materials and Methods}

\author{
Viruses, Animals, Biosafety, and Animal Care
}

Influenza viruses A/Puerto Rico/8/34, H1N1 (A/PR/8/34), and $\mathrm{A} / \mathrm{swine} / \mathrm{Iowa} / 15 / 30(\mathrm{~A} / \mathrm{SW} / 15 / 30)$ were obtained from ATCC (Manassas, VA). Viral titers were determined by median tissue culture infectivity dose $\left(\mathrm{TCID}_{50}\right)$ assay via infection of Madin-Darby canine kidney (ATCC) cells. ${ }^{18}$ Female BALB/c mice (6- to 8-week-old) were purchased from The Jackson Laboratory (Bar Harbor, ME). Mice were housed in micro-isolator cages in an Animal Biosafety Level 2 animal facility with food and water ad libitum. For pig influenza infections, 3- to 4-week-old piglets were obtained from a healthy herd at Oklahoma State University. Piglets were influenza-seronegative, tested in preserum via neutralization assay for absence of neutralizing antibodies against swine influenza $\mathrm{A} / \mathrm{SW} / 15 / 30$ virus and a pandemic isolate, swine $\mathrm{A} / \mathrm{New}$ York/18/2009 virus (randomly selected). ${ }^{39}$ Piglets from infection and infection plus treatment groups were housed in the same room, whereas mockinfected piglets were housed in a separate room. All piglet studies were performed in an Animal Biosafety Level 2 facility, in a negatively pressurized animal room with $100 \%$ high-efficiency particulate air filtered exhaust, at Oklahoma Animal Disease and Diagnostic Laboratory, with food and water ad libitum.

\section{Ethics Statement}

All mice (protocol number VM 17-32) and piglet (protocol number VM 16-5) studies were approved by the Institutional Animal Care and Use Committee of Oklahoma State University, and were performed in strict accordance with their recommendations. These experiments were conducted in accordance with the US Public Health Service Policy on Humane Care and Use of Laboratory Animals.

\section{Influenza Virus Infection}

Mice were anesthetized with a mixture of ketamine $(7.5 \mathrm{mg} /$ $\mathrm{kg})$ and xylazine $(0.1 \mathrm{mg} / \mathrm{kg})$ by i.p. injection. Mice were infected with lethal doses of A/PR/8/34 (2500 TCID $_{50}$ in 50 $\mu \mathrm{L}$ by intranasal route, equivalent to 10 median lethal dose, determined in our laboratory) diluted in phosphate-buffered saline (PBS). ${ }^{17}$ Equal volumes of PBS were inoculated into control mice.

Time-Dependent Kinetics of Neutrophil Influx, Activation, and NETosis in BAL Samples

Bronchoalveolar lavage (BAL) fluids were collected at 12 hours post infection (hpi) and 1, 2, 3, 4, 5, 6, 7, and 8 days post infection (dpi) from lethal influenza-challenged mice (five to six mice at each time point). For collection of BAL fluids, control and infected mice lungs were 
lavaged twice with intratracheal administrations of $0.5 \mathrm{~mL}$ of PBS, ${ }^{18}$ and the recovery of the BAL was $>90 \%$ for all animals. The BAL cells were separated, as described earlier, ${ }^{18}$ and differential cell counts were performed using modified Giemsa staining. Different cell types in BAL were identified (Supplemental Figure S1). One of the advantages of Giemsa method after cytospin is that it preserves exact cellular picture in BAL as there are no washing/resuspension steps involved. Cell-free BAL fluids were stored at $-80^{\circ} \mathrm{C}$ for determining MPO activity and neutrophil elastase (NE) levels. Whole lungs were fixed from mice infected at different time intervals, as described above, and histopathology analysis was performed (Table 1).

\section{Measurement of MPO Activity in BAL Fluids}

MPO activity in the BAL fluids collected from control and infected mice between 12 hpi and 8 dpi was determined, as described previously. ${ }^{20}$

\section{Enzyme-Linked Immunosorbent Assays for NE and NETs}

Levels of NE in BAL were measured using NE enzymelinked immunosorbent assay kit (R\&D Systems, Minneapolis, MN). The MPO/DNA enzyme-linked immunosorbent assay kit was used for measuring cell-free NETs, as described previously. ${ }^{21}$

\section{Immunostaining for $\mathrm{CitH} 3$ and $\mathrm{SP}-\mathrm{C}$}

For identifying NETs or surfactant protein C (SP-C; alveolar type II epithelial marker)-positive cells, BAL from control or infected mice at 12 hpi to $8 \mathrm{dpi}$ was stained with anti-citrullinated histone 3 (citH3) or anti-SP-C antibodies, respectively, followed by fluorescence-labeled secondary antibodies. At least 200 cells were counted in each sample to determine cells positive for citH3 and SP-C.
Time-Dependent Evaluation of Plasma CXCR2 Ligands in Lethal Influenza-Challenged Mice

CXCR2 ligands, including mouse keratinocyte chemoattractant/IL-8 and macrophage inflammatory protein, in the plasma samples of control or infected mice between 12 hpi and 8 dpi were evaluated by enzyme-linked immunosorbent assay (Roche, Indianapolis, IN), according to the manufacturer's instructions.

\section{SCH527123 and Oseltamivir Treatment in Lethal Influenza-Challenged Mice}

Murine lethal influenza (A/PR/8/34; $\left.2500 \mathrm{TCID}_{50}\right)$ infection model was used. Mice were divided into five groups: i) mock infected, ii) infected, iii) infected + oseltamivir treated; iv) infected + SCH527123 treated, and v) infected + oseltamivir + SCH527123 treated (Table 2). SCH527123 was administered alone or in combination with oseltamivir (equivalent to human dose), ${ }^{40}$ to assess the additive effects of SCH527123. Initial studies on dosage and timing of oseltamivir treatment indicated that administration of oseltamivir (30 mg/kg; Roche; orally using an oral gavage) starting at $24 \mathrm{hpi}$ or $48 \mathrm{hpi}$, followed by four additional doses at 1-day intervals did not yield any protection. However, oseltamivir treatment starting at $12 \mathrm{hpi}(20 \mathrm{mg} /$ $\mathrm{kg}$ ) followed by four additional dose treatments achieved about $30 \%$ survival in lethal influenza-challenged mice (Supplemental Figure S2). On the basis of these results, oseltamivir administered at 12 and $24 \mathrm{hpi}(10 \mathrm{mg} / \mathrm{kg})$ and at 2, 3, 4, and $5 \mathrm{dpi}(20 \mathrm{mg} / \mathrm{kg})$ was used throughout the experiments.

Treatment of SCH527123 was initiated at 2 dpi (neutrophils peak from $3 \mathrm{dpi}$ ) and continued until $6 \mathrm{dpi}$, using an oral gavage at a dose of $3 \mathrm{mg} / \mathrm{kg}$. Due to the primary goal of preventing massive neutrophil influx, SCH527123 was administered therapeutically, but not in a prophylactic manner. SCH527123 was prepared in 0.4\% methyl cellulose. ${ }^{40}$ Three different dosing regimens of SCH527123 were tested: three doses (2, 3, and $4 \mathrm{dpi})$, four doses $(2,3,4$, and $5 \mathrm{dpi})$, and five doses (2, 3, 4, 5, and $6 \mathrm{dpi})$. Clinical signs

Table 1 Semiquantitative Histopathology Scores

\begin{tabular}{|c|c|c|c|c|c|c|c|}
\hline Phase & Pathologic lesions & $\mathrm{CON}$ & $1 \mathrm{dpi}$ & $3 \mathrm{dpi}$ & $5 \mathrm{dpi}$ & $6 \mathrm{dpi}$ & $8 \mathrm{dpi}$ \\
\hline \multirow{2}{*}{ Inflammatory phase } & Bronchiolar inflammation & - & + & +++ & +++ & ++ & $++^{*}$ \\
\hline & Interstitial inflammation & - & + & +++ & ++++ & ++ & $++^{*}$ \\
\hline \multirow[t]{2}{*}{ Hemorrhagic exudative phase } & Hemorrhage & - & - & + & + & +++ & $++++{ }^{*}$ \\
\hline & Pulmonary edema & - & - & + & ++ & +++ & $++++^{*}$ \\
\hline
\end{tabular}

Blinded scoring (N.T.) of inflammatory phase and hemorrhagic exudative phase was evaluated by pathologic lesions, including necrotic bronchioles, bronchiolar inflammation, alveolitis, interstitial inflammation, pulmonary edema, hemorrhage, and fibrin deposition. $n=5$ mice per group.

$* P<0.01$ versus 3 dpi.

- , No lesions; + , minimum; ++ , mild; +++ , moderate; ++++ , severe; CON, control; dpi, days post infection. 
Table 2 Animal Groups from CON, Influenza Infection, and Treatment Groups

\begin{tabular}{llr}
\hline Group designation & Infection/treatment & Mice, $n$ \\
\hline CON & Phosphate-buffered saline & 10 \\
INF & Infection & 15 \\
INF + OSL & Infection and oseltamivir & 10 \\
INF + SCH & Infection and SCH527123 & 5 \\
INF + OSL + SCH (3 doses) & Infection, oseltamivir, and SCH527123 (3 doses) & 10 \\
INF + OSL + SCH (4 doses) & Infection, oseltamivir, and SCH527123 (4 doses) & 5 \\
INF + OSL + SCH (5 doses) & Infection, oseltamivir, and SCH527123 (5 doses) & 10 \\
\hline
\end{tabular}

The infection and treatment experiments were repeated at least twice, with five mice in each group per experiment. SCH527123 treatment did not show any protection, and was thus tested only once. The infection and treatment studies were repeated twice to collect bronchoalveolar lavage samples and lung tissues for histopathology.

CON, control.

(body weights, ruffled fur, difficulty in breathing, and hunched posture) were monitored for $21 \mathrm{dpi}$, and animals showing severe signs of difficulty in breathing, hunched posture, and weight loss were euthanized immediately.

\section{Collection of Tissues and BAL from Infected and Treatment Groups}

Lungs and BAL were collected from control and infected mice in which three doses of SCH527123 alone or in combination treatment groups were administered at $5 \mathrm{dpi}$. Protein leakage was determined by measuring total BAL protein content using a DC Protein Assay Kit (Bio-Rad, Irvine, CA).

\section{Histopathology Analysis}

Histopathologic analyses were performed, as described previously, and semiquantitative histopathologic scores were assigned in a blinded manner (T.A.S.), as described previously. ${ }^{21}$ The histopathologic lesions, including necrotizing bronchiolitis, inflammation in the bronchioles, alveolitis, interstitial inflammation, hemorrhage, edema, microvascular thrombosis, and endothelial damage, were scored on a 0 to 4 scale ( 0 for none or very minor, 1 for mild, 2 for intermediate, 3 for moderately severe, and 4 for severe and widespread), as described previously. ${ }^{21}$

\section{Swine Model of Influenza Infection}

Initially, a sublethal pig influenza infection model was established to evaluate neutrophil influx, NET induction, and lung pathogenic signatures. Piglets (3 to 4 weeks old) were anesthetized with intramuscular injection with ketamine $(33 \mathrm{mg} / \mathrm{kg})$ and xylazine $(2 \mathrm{mg} / \mathrm{kg})$ and infected with $10^{6} \mathrm{TCID}_{50}$ of the $\mathrm{A} / \mathrm{SW} / 15 / 30$ virus in $1 \mathrm{~mL}$ volume; 0.5 $\mathrm{mL}$ was inoculated into each nostril. Mock-infected piglets received equal volumes of PBS. ${ }^{41}$ Animals were monitored daily for clinical signs of rectal temperature and body weights and euthanized at 3 and 6 dpi (four piglets in each group).

\section{SCH527123 and Oseltamivir Treatment in Swine Influenza-Challenged Piglets}

Piglets were infected with $10^{6} \mathrm{TCID}_{50}$ of $\mathrm{A} / \mathrm{SW} / 15 / 30$, as described above. Five groups (four piglets per group), including: i) mock-infected, ii) infected, iii) infected + oseltamivir-treated, iv) infected + SCH527123-treated, and v) infected + oseltamivir + SCH527123-treated were included. Both oseltamivir and SCH537123 were given orally starting from 2 dpi to determine additive effect of SCH527123 (when combined as therapeutic treatment with antiviral agent) in reducing pulmonary pathology. A significant neutrophil influx was observed at $3 \mathrm{dpi}$ in infected piglets. Animals were treated with oseltamivir $(5 \mathrm{mg} / \mathrm{kg})$ or SCH527123 $(0.5 \mathrm{mg} / \mathrm{kg})$ alone or in combination. Clinical signs were monitored as described above. Measurement of physical activity during infection and treatments was challenging as the piglets quickly become alert. Animals were euthanized at $5 \mathrm{dpi}$. One piglet from $\mathrm{SCH}$ group died during anesthesia, and was excluded from this study.

\section{Gross and Microscopic Pathologic Examination and Viral Antigen Detection}

For euthanasia, piglets from control, infected, and treatment groups were first anesthetized with ketamine/xylazine, and blood was collected from medial saphenous vein, followed by intravenous pentobarbital (Beuthanasia D; Virbac AH Inc., Fort Worth, TX; $390 \mathrm{mg}$ per 10 pounds) injection. At necropsy, gross lesions were examined and images were taken without excising the lungs (to prevent any blood contamination during lavage collection or formalin fixing). ${ }^{20}$ Right lung lobes were clamped, whereas left lung lobes were lavaged twice with $30 \mathrm{~mL}$ of sterile PBS. Following lavage, left lung lobes were clamped and right lobes were fixed with $4 \%$ neutral-buffered formalin. For microscopic examination, cranial, middle, and caudal lobes (randomly selected $0.5-$ to $1-\mathrm{cm}$ slices from each lobe) were embedded in paraffin. Sections $(6 \mu \mathrm{m}$ thick) were cut and stained with hematoxylin and eosin. The lung tissues were scored in a blinded manner (T.A.S.) for necrotizing 
bronchiolitis, alveolitis, inflammation, edema, microvascular thrombosis, pulmonary hemorrhage, and endothelial damage, ranging from 0 (no pathologic lesions) to 4 (severe lung pathology).

Next, distribution of viral antigen was determined in different lung lobes of piglets, which were used for histopathology analyses. Lung sections were probed with swine influenza virus-specific anti-matrix 1 protein antibody (GeneTex, Irvine, CA), followed by secondary horseradish peroxidase conjugated antibodies. 3,3',5,5'-tetramethylbenzidine (TMB) substrate was used for color development. For semiquantitative analysis, influenza-positive scores were calculated by estimating the number of antigen-positive cells per $\times 200$ microscopic field. The scores were assigned, as described previously, ${ }^{42}$ using the following criteria: 0 indicates no positive cells; 1 , minor ( $<10$ positive cells); 2 , moderate (11 to 25 positive cells); and 3, high ( $>26$ positive cells).

\section{Western Blot Analysis}

BAL fluids were analyzed for extracellular histones (H2A or H2B; Millipore, Danvers, MA) and modified histones (citH3 or citH4; Millipore) by Western blot analysis, as described previously. ${ }^{20}$ Lung injury was assessed by $\mathrm{T} 1-\alpha$ (a plasma membrane protein expressed on alveolar type I pneumocytes) and claudin 5 (a tight junction protein in endothelial cells) by Western blot analysis. ${ }^{20}$ All of the Western blots were subjected to ImageJ analysis (ImageJ with 64-bit Java 1.8.0_172; NIH, Bethesda, MD; https://imagej.nih.gov/ij) for performing densitometry measurements.

\section{Determination of the Virus Titers in Mice by $\mathrm{TCID}_{50}$ Assay}

The virus titers in the murine lung homogenates from control, infected, and treatment groups (three doses of SCH527123) at 5 dpi were determined by TCID $_{50}$ assay, as described previously. ${ }^{1}$ Madin-Darby canine kidney cells were infected with serial 10 -fold dilutions of lung homogenates from control, infected, and treatment groups. Known 10 -fold dilutions of influenza PR/8/34 virus also served as the positive control for titer determination. At 72 hours hpi, Madin-Darby canine kidney cells were examined for cytopathic effect, and virus titers were calculated according to the Reed-Muench method. ${ }^{43}$ To test whether SCH527123 exhibits any antiviral activity, 10-fold serial dilutions of SCH527123 (1 $\mathrm{mmol} / \mathrm{L}$ to $10 \mathrm{nmol} / \mathrm{L})$ were added to Madin-Darby canine kidney cells infected with virus at multiplicity of infection of 1 . Cytopathic effect of the virus infection was monitored at $72 \mathrm{hpi}$, analyzed by TCID $_{50}$ assay, and expressed as $\mathrm{TCID}_{50}$ per $\mathrm{mg}$ of lung protein.

\section{In Vitro NET Induction and Inhibition Studies}

Blood neutrophils were isolated from control and infected mice using MACS neutrophil isolation kit (Miltenyi Biotec
Inc., Auburn, CA) at $3 \mathrm{dpi}$, as described previously. ${ }^{21} \mathrm{Pu}-$ rified neutrophils were stimulated with IL-8 $(100 \mathrm{ng} / \mathrm{mL})$ for 3 hours. For NET inhibition studies, neutrophils from influenza-infected mice were stimulated with IL-8 in the presence or absence of anti-CXCR2 $(10 \mu \mathrm{g} / \mathrm{mL})$ or anti-IL$8(10 \mu \mathrm{g} / \mathrm{mL})$ antibodies or anti-mouse $\operatorname{IgG}(10 \mu \mathrm{g} / \mathrm{mL})$ or SCH527123 $(2 \mu \mathrm{g} / \mathrm{mL})$. Phorbol 12-myristate 13-acetate at $20 \mathrm{nmol} / \mathrm{L}$ concentration was used as a positive control. Cells were stained with Sytox green to detect NETs. ${ }^{20}$

\section{NE Nuclear Translocation}

To determine the effect of CXCR2 signaling on NE nuclear translocation, IL-8 stimulated or unstimulated neutrophils were stained with anti-NE and anti-MPO antibodies and counterstained with DNA dye, DAPI. Quantification of NE translocation was evaluated by NE colocalized with MPO (marker for $\alpha$ granules) versus NE colocalization with DAPI, by randomly selecting 15 to 20 cells $(1000 \times)$ using the Olympus fluorescence microscope (Olympus, Waltham, MA). ${ }^{44}$

\section{CXCR2 Stimulation on Citrullination of Histones and Role of Oxidation-Reduction Enzymes}

Neutrophils were incubated with or without inhibitors of NADPH (diphenyleneiodonium), MPO (4-aminobenzoic acid hydrazide), or CXCR2 antagonist (SCH527123). Citrullination of histones was determined by immunostaining with anti-citH 3 antibody, and quantified by counting at least 100 cells per slide.

\section{Signaling Pathways in IL-8-Induced ROS Generation}

Reactive oxygen species (ROS) generation is critical in enhancing oxidation-reduction enzyme activities during NET release. To test the downstream signaling in CXCR2mediated ROS generation, neutrophils were stimulated with IL-8 in the presence of inhibitors of protein kinase C/ G06983, $5 \mu \mathrm{mol} / \mathrm{L}$; mechanistic target of rapamycin (mTOR) inhibitor/2,4-dinitrophenol, $100 \mu \mathrm{mol} / \mathrm{L}$; extracellular signal-regulated kinase (ERK) inhibitor/SCH772984, $10 \mu \mathrm{mol} / \mathrm{L}$; and p38 mitogen-activated protein kinase inhibitor/SB203580, $10 \mu \mathrm{mol} / \mathrm{L}$. ROS released was measured by using $2^{\prime}-7^{\prime}$-dichlorodihydrofluorecein diacetate by excitation and emission readings at 430 and $510 \mathrm{~nm}^{8}$

\section{Effect of ERK Signaling on CXCR2-Induced NETosis}

To test whether blocking ERK signaling inhibits citrullination process during NETosis, neutrophils were stimulated with IL-8 in the presence of ERK inhibitor (SCH772984; 10 $\mu \mathrm{mol} / \mathrm{L})$ or mTOR inhibitor (2,4-dinitrophenol; 100 $\mu \mathrm{mol} / \mathrm{L})$. Citrullination of histones was measured as described above. 


\section{Statistical Analysis}

Statistical analyses were performed by analysis of variance or $t$-test. Animal treatment and survival curve comparisons were analyzed by the Mantel-Cox log-rank test. For temporal analysis and other comparison studies, one-way analysis of variance and the Tukey multiple comparison tests were used $(P<0.05)$, or the $t$-test was used for comparison between two groups. All statistical analyses were performed using Excel or GraphPad Prism 7 software (GraphPad, San Diego, CA). Data are expressed as mean \pm SEM. $P<0.05$ is considered statistically significant.

\section{Results}

Temporal Kinetics of Neutrophil Recruitment, Activation, and NET Release in Correlation with the Levels of CXCR2 Ligands and Lung Pathogenic Signature in Lethal Influenza-Challenged Mice

To evaluate temporal kinetics of neutrophil influx, their activation, and NETosis, BAL samples were collected from control mice or from lethal influenza-challenged mice at 12 hpi and at 1, 2, 3, 4, 5, 6, 7, and 8 dpi. The time-dependent changes in BAL cellular signature are illustrated in Supplemental Figure S1. On infection, significant increase in neutrophils was observed by $12 \mathrm{hpi}$, peaked at $3 \mathrm{dpi}$, remained high until $6 \mathrm{dpi}$, and declined thereafter (Figure 1A). MPO activities in cell-free BAL fluids exhibited similar trends, with the neutrophil sequestrations in infected lungs (Figure 1B). Analysis of variance and post-hoc analysis with Tukey multiple comparison tests were performed to compare different time points. NET formation (measured by citH3 staining) was significantly increased between 2 and 6 dpi in parallel with neutrophil influx and MPO activities (Figure 1, C and D). The timedependent changes in neutrophil activity and NETs correlated with elevated plasma levels of CXCR2 ligands, including mouse keratinocyte chemoattractant (Figure 1E) and macrophage inflammatory protein (Supplemental Figure S3A). The increase in neutrophil activity and NETs between 3 and 5 dpi directly correlated with alveolar epithelial injury (indicated by the presence of SP-C, a type II alveolar epithelial marker) in the BAL (Figure $1 \mathrm{~F}$ and Supplemental Figure S3B), and with pathologic lesions of aggravated necrotizing bronchiolitis, alveolar damage, and interstitial inflammation in infected lungs (Table 1). Interestingly, neutrophil activity and NETs declined by $7 \mathrm{dpi}$, by which time infected lungs displayed widespread hemorrhage and vascular leakage (Supplemental Figure S1 and Table 1). These findings reveal that dynamics of neutrophils and NETs significantly alter during infection, and that early immunopathologic events may play detrimental role in subsequent vascular injury and respiratory failure in severe influenza pneumonia.
Administration of SCH527123 in Combination with Oseltamivir Protects Mice from Lethal Influenza Infection

On the basis of the temporal dynamics of neutrophil activity and NETosis, the efficacies of SCH527123 alone or together with oseltamivir were tested in a murine model of lethal influenza pneumonia. ${ }^{19}$ All infected mice succumbed to infection between 6 and $8 \mathrm{dpi}$, with a mean survival time of $6.5 \pm 2$ days (Figure 2). Administration of oseltamivir monotherapy resulted in about $15 \%$ survival rate, with the mean survival time increasing to $11 \pm 2$ days. Administration of SCH527123 alone did not confer any protection, and all treated mice died by 8 dpi. However, administration of SCH527123 plus oseltamivir significantly protected from lethal influenza. Animals treated with three doses of SCH527123 plus oseltamivir achieved $80 \%$ survival. All animals treated with four doses of SCH527123 plus oseltamivir survived (Figure 2A). Treatment with five doses of SCH527123 together with oseltamivir conferred 70\% survival. Survival curves of infected animals from different treatment groups were analyzed by the Mantel-Cox log-rank test. In combination treatment groups, mortalities of mice were observed after $12 \mathrm{dpi}$, with mean survival duration of 19 to 21 dpi (Figure 2B). Body weights of control, infected, and treated mice were monitored daily (Supplemental Figure S4). All of the recovering animals regained normal physical activities, including eating, and drinking.

\section{Administration of SCH527123 plus Oseltamivir Decreases Lung Pathology, NETs, and ECH Release}

To investigate the protective mechanisms of SCH527123 in the combination treatment, animals from mock-infected, virusinfected, and monotherapy or combination therapy were analyzed at three-dose SCH527123 treatment groups for lung pathology, NET release, and accumulation of ECH at 5 dpi. Mice with infection alone exhibited widespread alveolitis and extensive neutrophilic influx. Severe bronchiolitis was evident by massive inflammatory cellular cuffing around bronchioles (Figure 3A). Although infected mice treated with SCH527123 alone exhibited relatively less inflammation, there was extensive airway and alveolar epithelial injury, likely due to a virusinflicted cytopathic effect. Infected mice given oseltamivir alone also displayed severe lung injury. In contrast, administration of SCH527123 plus oseltamivir in infected mice significantly reduced alveolar injury, edema, pulmonary hemorrhage, and inflammation (Figure 3A). Semiquantitative histopathologic analysis revealed significant reduction in lung pathology in the combination treatment group compared with treatment with either drug alone (Figure 3B). Furthermore, inclusion of SCH527123 in the combination regimen also significantly reduced $\mathrm{H} 2 \mathrm{~A}$ and citH3 levels, compared with treatment with either drug alone (Figure 3, C and D). Similarly, combination treatment reduced NET release (Figure 3E). 
A

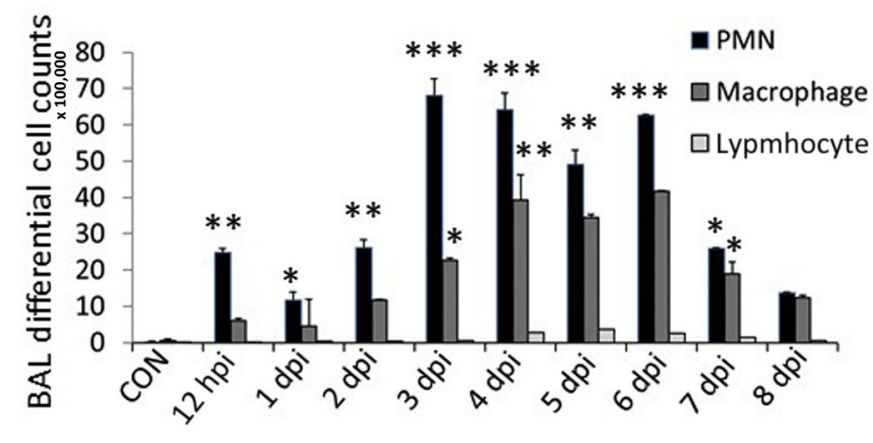

C
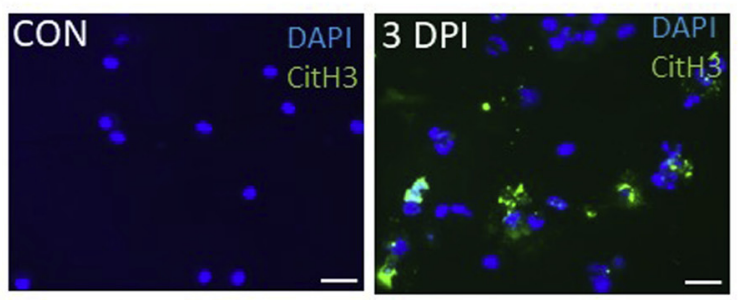

E

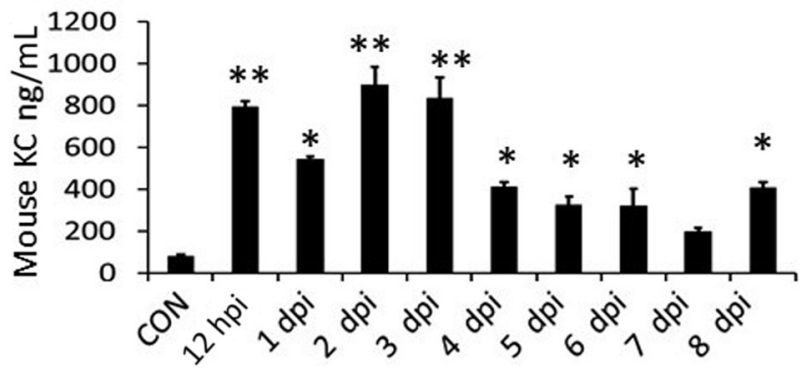

B

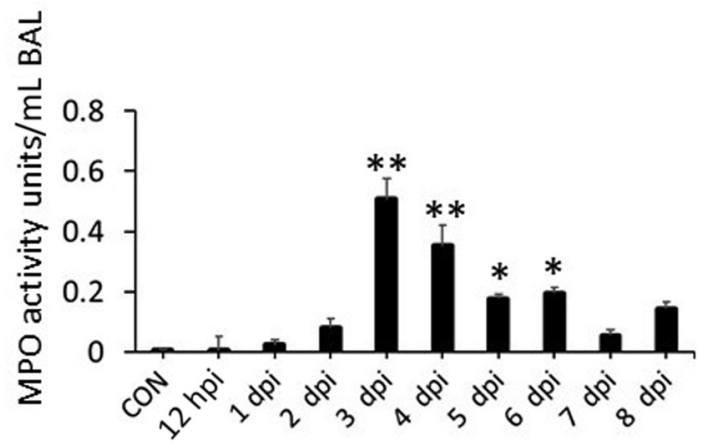

D

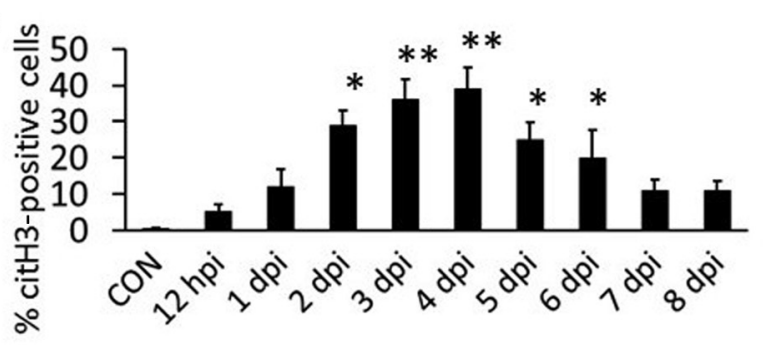

$\mathbf{F}$

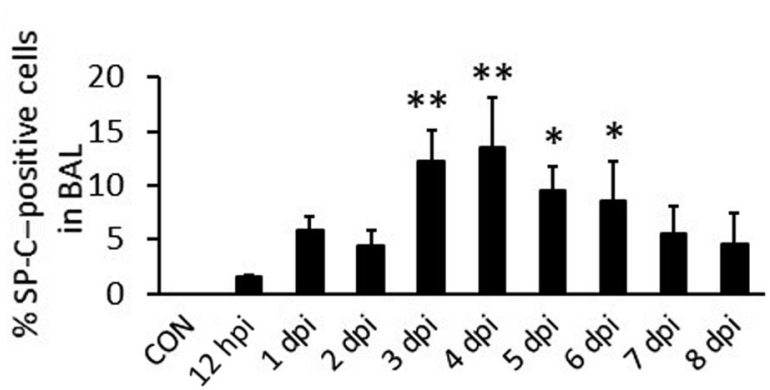

Figure 1 Temporal kinetics of neutrophil sequestration, activation, NETosis, and levels of chemokine ligands in lethal influenza infection. BALB/c mice were challenged with 2500 median tissue culture infectivity dose of influenza A/PR/8 virus. A: Differential cell counts of bronchoalveolar lavage (BAL) samples were measured by modified Giemsa staining between 0 hours post infection [hpi; control (CON)] and 8 days post infection (dpi). Neutrophil-dominant infiltration was observed at all time points, with peak neutrophil influx between 3 and 6 dpi. Cellular infiltrates were drastically reduced from 7 dpi. B: Relative myeloperoxidase (MPO) activity in cell-free BAL fluids from control and infected mice between $12 \mathrm{hpi}$ and 8 dpi. C and D: Positive citrullinated histone 3 (citH3) staining in BAL cells from control and infected mice. DAPI was the nuclear dye. E: Mouse keratinocyte chemoattractant (KC) in plasma samples collected at different time intervals during influenza infection. F: Epithelial damage was evaluated by the presence of dislodged alveolar epithelial cells in BAL, and by immunostaining for surfactant protein $\mathrm{C}(\mathrm{SP}-\mathrm{C})$ in a time-dependent manner. One-way analysis of variance and post-hoc analysis with Tukey multiple comparison tests were performed to compare different time points. Results are expressed as means \pm SEM (A, B, and $\mathbf{D}-\mathbf{F})$. $n=4$ to 5 mice per group $(\mathbf{A}, \mathbf{B}$, and $\mathbf{D}-\mathbf{F}) .{ }^{*} P<0.05,{ }^{*} P<0.01$, and ${ }^{*}{ }^{*} P<0.001$ versus control. Scale bars $=40 \mu \mathrm{m}$ (C). PMN, neutrophils.

\section{Combination Treatment Decreases Inflammation,} Alveolar-Capillary Damage, and Vascular Leakage

Massive neutrophil influx and widespread alveolitis are major pathologic events that drive vascular leakage and respiratory failure in severe influenza infections. ${ }^{17-19}$ Administration of SCH527123 alone or in combination with oseltamivir significantly reduced neutrophil influx (Figure 4A). Combination therapy significantly reduced alveolar-capillary damage, as evident by decreased T1- $\alpha$ and claudin 5 levels in BAL samples compared with treatment with either drug alone (Figure 4, B and C). Similarly, significantly decreased total proteins in BAL samples were observed in combination-treated animals compared with untreated infected mice or other single treatment groups (Supplemental Figure S5A). Decreased lung viral loads were observed in infected mice with oseltamivir treatment alone and with combination treatment (Supplemental Figure S5B).

Therapeutic Administration of SCH527123 plus Oseltamivir Attenuates Gross Pathologic Lung Lesions in Piglets Infected with Swine Influenza Virus

Like humans, pigs are natural hosts for influenza and share many similarities in lung pathology, immune responses, and distribution of viral receptors in the lungs. Therefore, pigs 
are considered as excellent surrogate models to understand human influenza infections. ${ }^{45-47}$ Piglets were challenged with $\mathrm{A} / \mathrm{SW} / 15 / 30$ virus at a dose that causes mild-tomoderate lung pathology. The gross lung pathology appeared as multifocal reddish-tan consolidation in all lung lobes (Figure 5A). Administration of SCH527123 alone did not significantly reduce gross pathologic lesions. Oseltamivir monotherapy relatively reduced lung gross lesions compared with the untreated infected animal group (Figure 5B). In comparison, SCH527123 plus oseltamivir treatment significantly diminished gross pathologic lesions compared with treatment with either drug alone. All infected piglets exhibited typical clinical signs of influenza with nasal secretions. There were no obvious differences in rectal temperatures or body weights among infected and treatment groups at this viral challenge dose (Figure 5, C and D).

\section{Combination Treatment Decreases Lung Pathology, NETs, and Viral Antigen Scores in Piglets}

Microscopic pathologic lesions evaluated in the cranial, middle, and caudal lung lobes revealed significant alveolitis and interstitial inflammation with neutrophil-specific cuffing in infected piglets (Figure 6A). Damaged areas of the lungs displayed necrotic epithelium with frequent presence of red blood cells within the alveoli. Monotherapy with either oseltamivir or SCH527123 alone showed decreased interstitial inflammation, which was not significant compared with the infected and untreated groups. However, combination treatment significantly restored normal lung architecture (Figure 6B), suggesting that inclusion of SCH527123 may serve as a potential complementary therapy to mitigate pulmonary damage in influenza infections. Co-administration of SCH527123 and oseltamivir also diminished the release of ECH and NETs compared with other treatment groups (Figure 6, C and D). In addition, piglets receiving the drug combination also displayed markedly diminished viral antigen scores compared with other groups (Figure 6, E and F).

\section{CXCR2 Stimulation Induces NETosis via Augmented Expression and Nuclear Translocation of NE}

Stimulation of CXCR2 with its ligand IL- 8 induces NETs during influenza. ${ }^{26}$ To test whether influenza infection enhances susceptibility of neutrophils toward NETosis, circulating neutrophils isolated from mock-infected or influenza-infected mice were stimulated with IL-8. Indeed, IL-8 stimulation significantly induced NET release from neutrophils isolated from influenza-infected mice (Figure 7A). Furthermore, anti-IL-8 antibody or competitive inhibition by SCH527123 or anti-CXCR2 antibody significantly diminished NETosis (Figure 7, B and C). NE nuclear translocation is essential for histone breakdown during NET formation. CXCR2 stimulation triggered significant NE nuclear translocation, assessed by

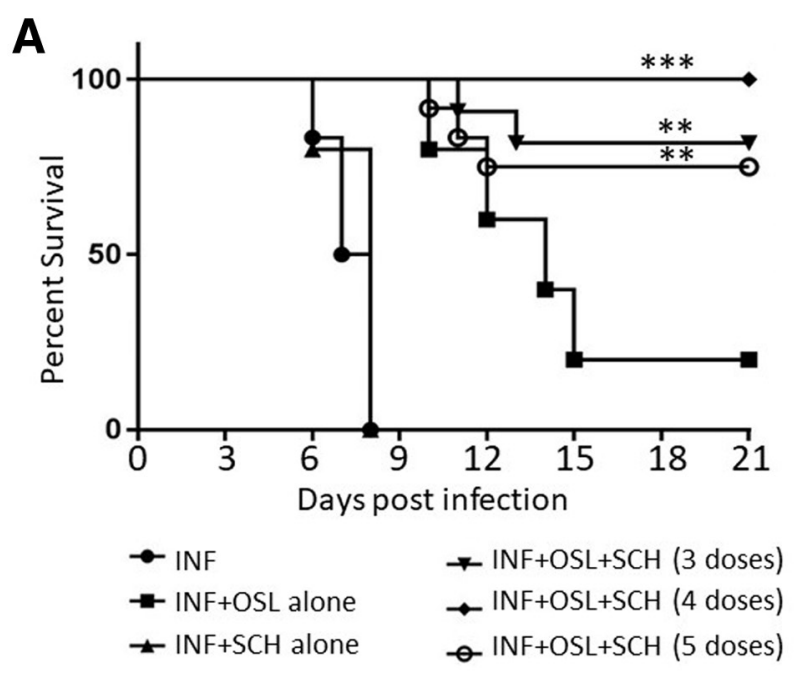

B

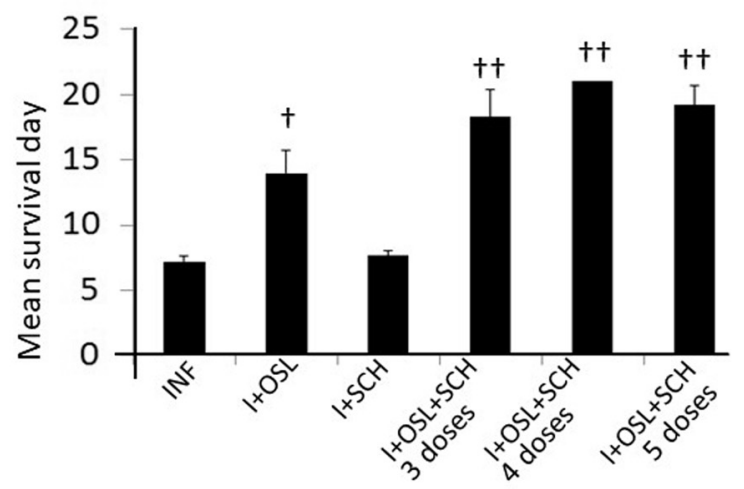

Figure 2 SCH527123 (SCH) when combined with oseltamivir (OSL) confers significant protection against lethal influenza challenge in mice. BALB/C mice were infected with 2500 median tissue culture infectivity dose of influenza A/PR/8 virus. A: Survival of the groups of infected mice receiving different drug treatments was monitored until 21 days post infection (dpi). All mice in the infected group (INF or I) and the SCH treatment alone group died by 7 and 9 dpi, respectively. Administration of OSL alone resulted in about $15 \%$ survival. The addition of SCH together with OSL achieved high survival rates. $\mathrm{SCH}$ was tested by administering three different regimens (ie, three, four, and five doses, as described). B: Mean days of survival of mice from influenza-infected and treatment groups. Animal treatment and survival curve comparisons were analyzed by the Mantel-Cox log-rank test with $95 \%$ CI. Results are expressed as means \pm SEM (B). $n=15$ in INF group (B); $n=10$ in OSL and OSL + SCH (three and five doses) groups (B); $n=5$ in OSL + SCH (four doses) and SCH alone groups (B). ${ }^{* *} P<0.01,{ }^{* * *} P<0.001$ versus $\mathrm{I}+0 \mathrm{SL} ;{ }^{\dagger} P<0.05$, ${ }^{\dagger} P<0.01$ versus INF group.

immunofluorescence microscopy. Unstimulated neutrophils showed colocalization of MPO and NE, indicating azurophilic granule staining (Figure 7, D and E). IL8-stimulated cells also exhibited decondensed nuclei on DAPI staining (Figure 7D). IL-8-induced NE nuclear translocation was significantly inhibited by the MPO inhibitor (4-aminobenzoic acid hydrazide) (Supplemental Figure S6). However, blocking MPO did not alter NE expression (Figure 7F). 


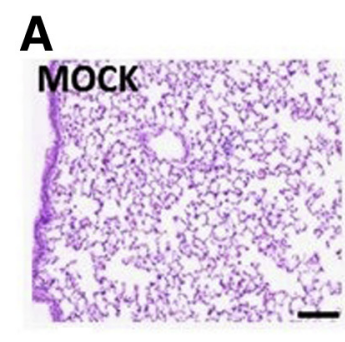

B

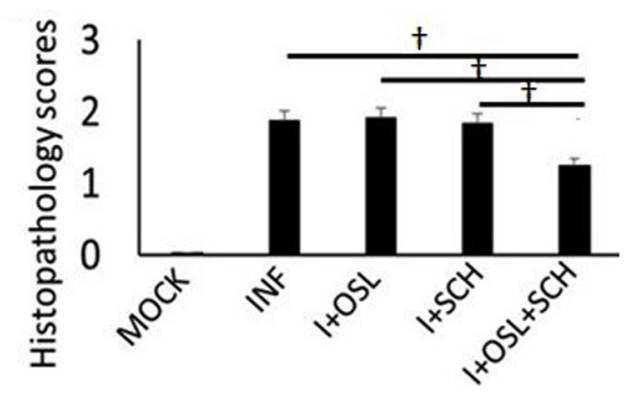

D

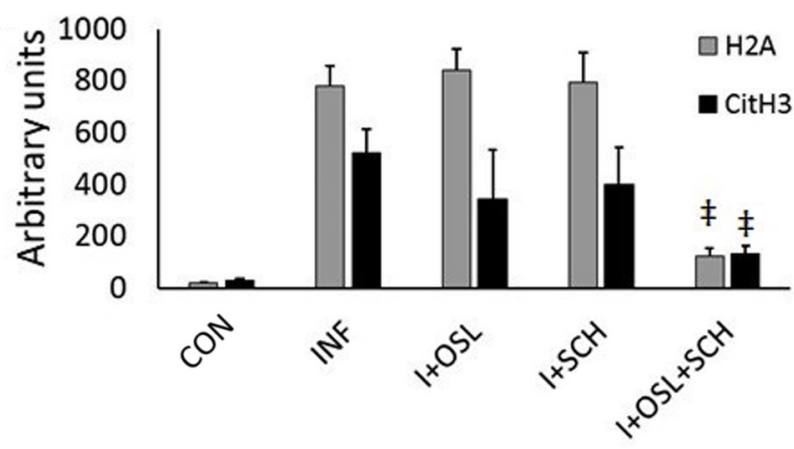

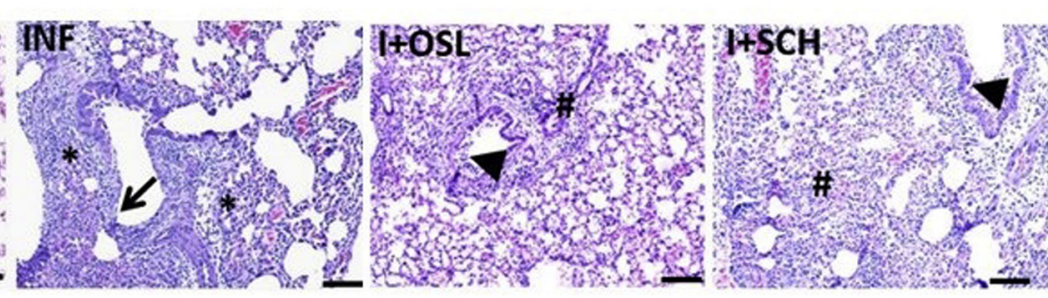

$1+$ OSK + SCH

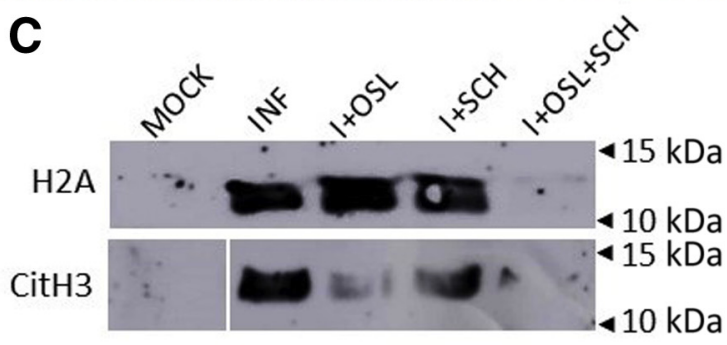

E

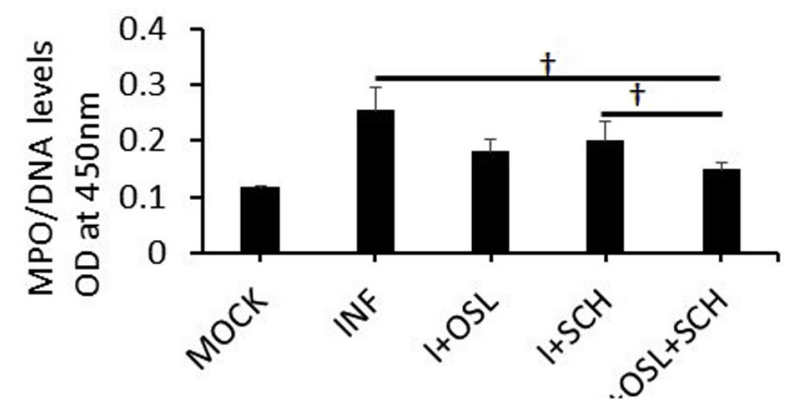

Figure $3 \mathrm{SCH} 527123(\mathrm{SCH})$ plus oseltamivir (OSL) reduce lung pathology and NETosis in lethal influenza-challenged mice. Animals from the groups of mock infection (MOCK), infection with monotherapy (INF or I), or infection with combination therapy (at three SCH dose regimen) were subjected to pathologic and biochemical analyses at 5 days post infection. A: Paraffin-embedded lung tissues from mock-infected mice, influenza-infected mice, and drug-treated mice were stained with hematoxylin and eosin. Histopathologic analysis of infected and untreated mice displayed bronchopneumonia (arrow) and severe alveolitis (asterisks). Infected mice treated with $\mathrm{OSL}$ alone $(\mathrm{I}+\mathrm{OSL}$ ) and SCH alone $(\mathrm{I}+\mathrm{SCH})$ also displayed extensive bronchiolar necrosis (arrowheads) and disrupted alveoli (hash marks). Mice in the combination treatment group ( $+\mathrm{SCH}+\mathrm{OSL}$ ) revealed significant diminution in lung pathology compared with other treatment groups. No pathologic lesions were found in mock-infected mice. B: Semiquantitative analysis of histopathologic scores of lung sections from mockinfected, influenza-infected, and treatment groups. C and D: Western blot analyses and their densitometry analyses for H2A and citrullinated histone 3 (citH3), performed on bronchoalveolar lavage samples of mock-infected, influenza-infected, and treatment groups. E: NET release into the plasma by measuring myeloperoxidase (MP0)/DNA levels by enzyme-linked immunosorbent assay. One-way analysis of variance and Tukey multiple comparison tests were performed. Overall analysis of variance was $P<0.05$. INF versus either drug alone or combination treatment was used for histopathology analysis. Densitometric data for $\mathrm{H} 2 \mathrm{~A}$, citH3, and MP0/DNA levels were analyzed by $t$-test between infected and treatment groups. Results are expressed as means \pm SEM $(\mathbf{A}, \mathbf{D}$, and $\mathbf{E}) . n=5$ mice per group (A); $n=4$ to 5 mice per group (E). ${ }^{\dagger} P<0.05 ; \ddagger P<0.05$ versus INF and monotherapy groups. Scale bars $=20 \mu \mathrm{m}(\mathbf{A})$. CON, control.

\section{CXCR2-Induced NETosis Is Dependent on ERK-Mediated ROS Generation}

Next, the downstream regulation of CXCR2-induced NETosis was investigated. IL-8 significantly enhanced citrullination of histones (Figure 8, A and B); the latter was inhibited by treatment with SCH527123, diphenyleneiodonium (NADPH oxidase inhibitor that blocks ROS generation), or MPO inhibitor, suggesting that CXCR2mediated NETosis is regulated by ROS generation (Figure 8C). To further investigate the downstream signaling activation involved in CXCR2-mediated ROS generation, neutrophils were stimulated with IL-8 in the presence of inhibitors of protein kinase C, mTOR, ERK, or p38 signaling pathways, and ROS generation was measured by flow cytometry. Inhibition of the ERK pathway decreased $70 \%$ of ROS generation (Figure 8D). Next, we evaluated whether blocking ERK signaling could inhibit IL-8-induced NETosis. Neutrophils were stimulated with IL-8 with or without ERK or mTOR inhibitors to evaluate citrullination of histones. Inhibiting ERK significantly decreased citH3 staining, indicating that CXCR2-induced ROS generation is mediated via induction of ERK (Figure 8E). 

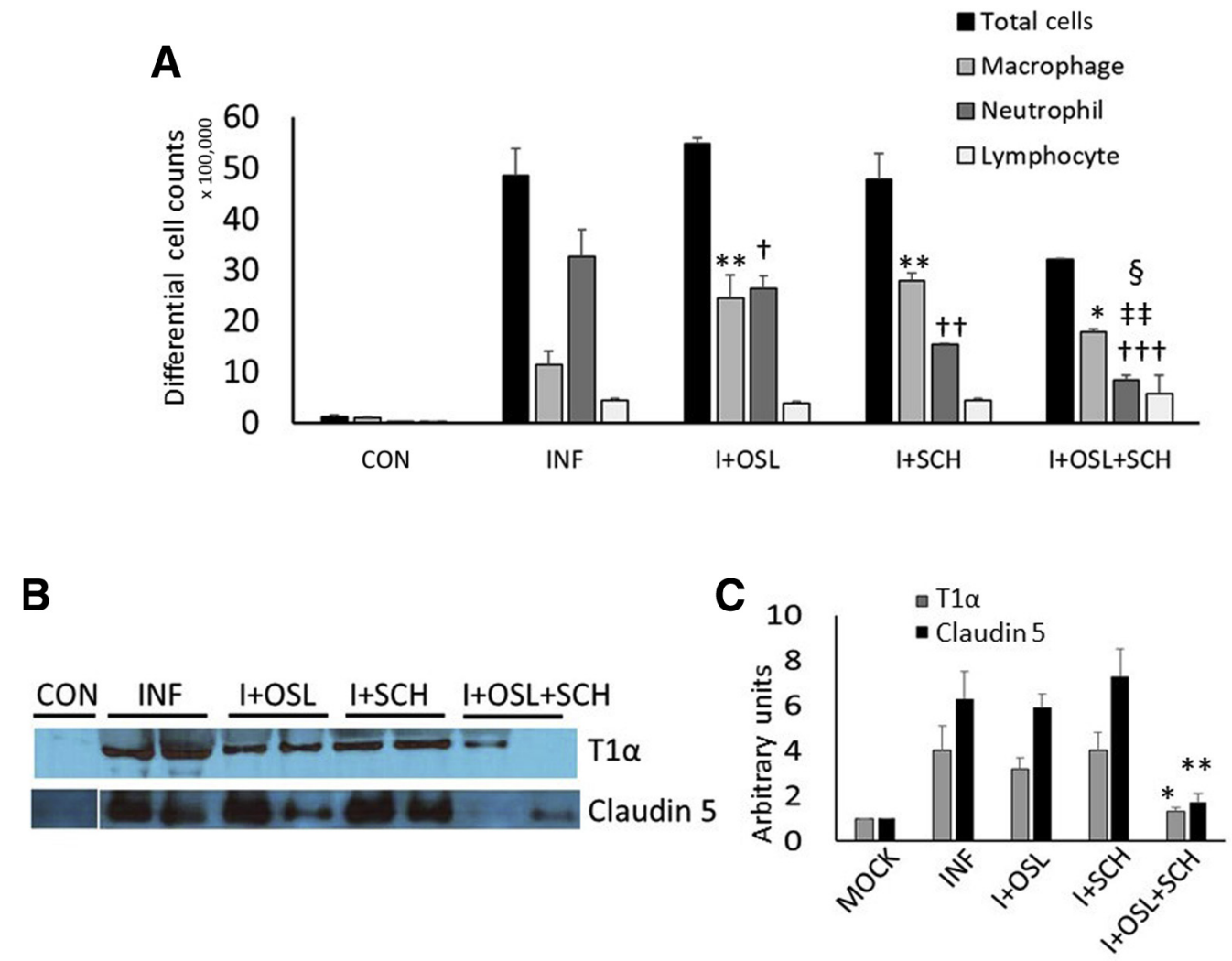

Figure 4 SCH527123 (SCH) plus oseltamivir (OSL) decrease inflammation, alveolar-capillary damage, and vascular leakage in lethal influenza-challenged mice. Animals from groups of mock infection (MOCK), infection (INF or I), monotherapy, or combination therapy (at three-dose SCH treatment regimen) were analyzed for alveolar-capillary damage and vascular leakage at 5 days post infection. A: Total and differential cell counts in bronchoalveolar lavage samples were measured by modified Giemsa staining. B: Administration of SCH527123 plus oseltamivir significantly inhibits alveolar-capillary damage in the lungs, as evident by decreased levels of T1- $\alpha$ and claudin 5 markers for alveolar type I epithelium and endothelium, respectively. C: Densitometric analysis. One-way analysis of variance and Tukey multiple comparison tests, were performed. Overall analysis of variance was $P<0.05$. Results are expressed as means \pm SEM ( $A$ and $\mathbf{C}$ ). $n=4$ to 5 mice per group (A and $\mathbf{C}$ ). ${ }^{*} P<0.05,{ }^{*} P<0.01$ versus INF group (macrophages); ${ }^{\dagger} P<0.05,{ }^{\dagger \dagger} P<0.01$, and ${ }^{\dagger \dagger \dagger} P<0.001$ versus INF group; ${ }^{\ddagger \ddagger} P<0.01$ versus I + OSL group; ${ }^{\S} P<0.05$ versus I + SCH group (neutrophils). CON, control.

\section{Discussion}

Although antiviral drugs represent an effective treatment modality against influenza, the mortality rates remain high in hospitalized patients with severe influenza pneumonia. $2,5,48,49$ Poor clinical outcomes from antiviral treatment have been attributed to the delay in initiation of antiviral therapy or to bystander effects of exaggerated host inflammatory immune responses. ${ }^{13-15}$ Herein, we demonstrate that treatment with SCH527123 plus oseltamivir improved survival in mice challenged with a lethal dose of influenza virus, and attenuated pulmonary pathology in piglets with sublethal swine influenza infection.

Overwhelming neutrophil activation and accumulation of NETs are associated with disease severity in patients with fatal influenza infections. ${ }^{24,25}$ Previously, we reported that NET components, such as ECH, neutrophil elastase, and matrix metalloproteinase 9, cause alveolitis and vascular pathology by disrupting the alveolar-capillary barrier. ${ }^{18,20,49}$ Currently, there are no clinically available drugs that target NET formation or exaggerated neutrophil activity.
Numerous studies on experimental blocking of CXCR2 (a major signaling receptor involved in neutrophil recruitment and activity) have shown ameliorative effects on lung injury and inflammatory responses in murine models of sublethal influenza. ${ }^{50,51}$ However, phase 2 clinical trials have shown that treatment with danirixin, a CXCR2 antagonist, does not influence clinical outcomes in influenza-infected patients when administered together with oseltamivir, compared with those administered oseltamivir alone. ${ }^{27,28}$ These clinical studies do not provide information as to the best time to administer danirixin to suppress excessive neutrophil influx, given that sufficient numbers of neutrophils are also important in host defense. ${ }^{52}$

In this study, we found a strong correlation between temporal dynamics of neutrophil influx and NET release with changes in lung pathology in lethal influenza-infected mice. These temporal analyses led to the identification of two distinctive phases in lung pathologies. The inflammatory phase occurred between 3 and $5 \mathrm{dpi}$, which represents an early phase of virus infection characterized by massive neutrophil influx, increased NETosis, and elevated MPO 
A

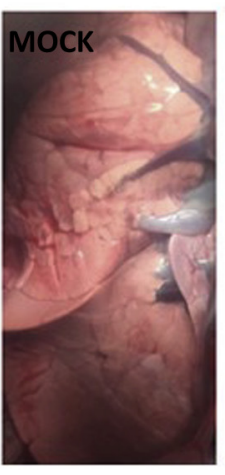

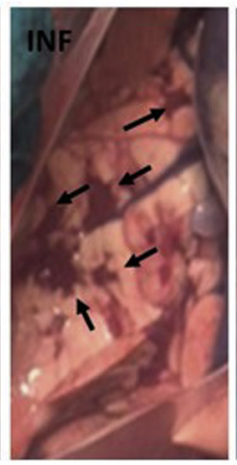
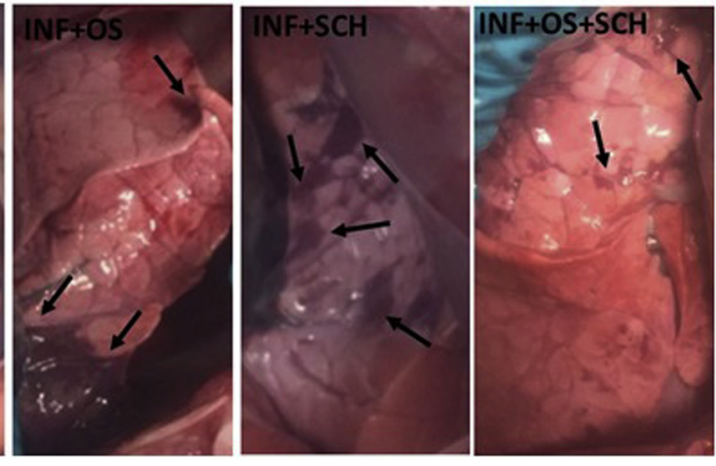

B

C

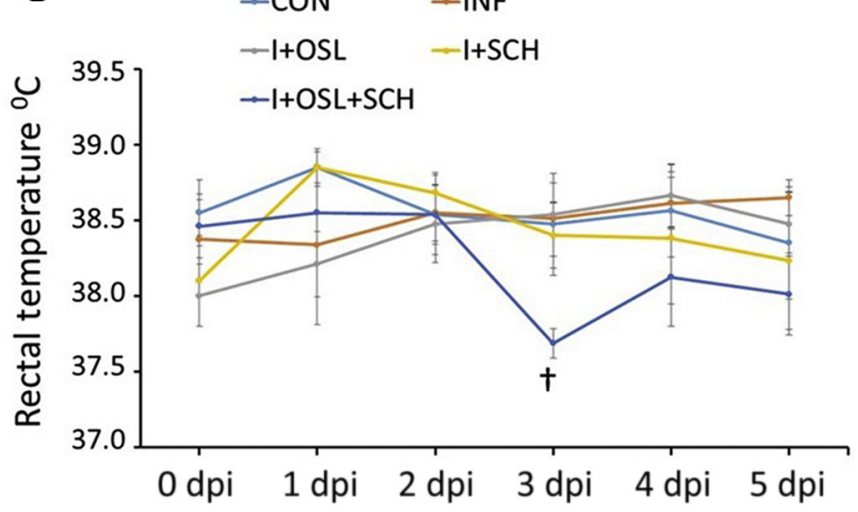

D

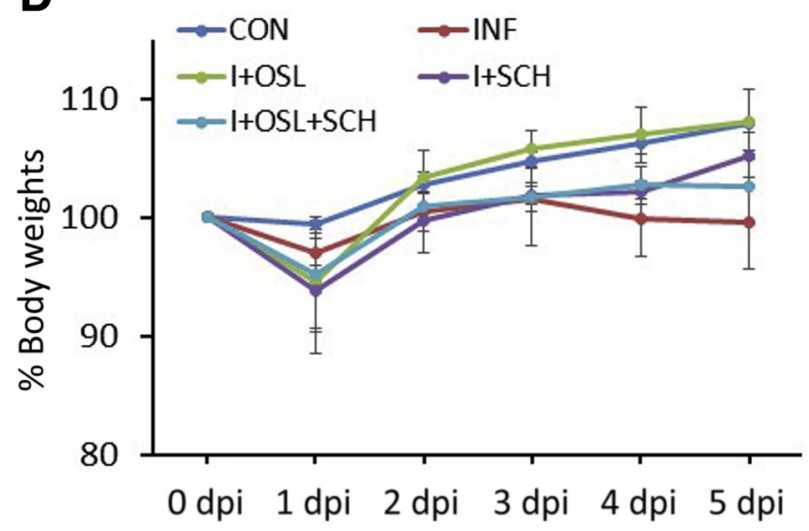

Figure $5 \mathrm{SCH} 527123$ (SCH) plus oseltamivir (OSL) reduce gross pulmonary lesions in piglets infected with swine influenza virus. Piglets ( 3 to 4 weeks old) were infected intranasally with swine influenza virus (A/swine/Iowa/15/30; SIV) at $1 \times 10^{6}$ median tissue culture infectivity dose. Piglets were treated with oseltamivir or SCH527123 alone or in combination, and euthanized at 5 days post infection (dpi). A: The dark red hemorrhagic consolidated lung lesions (arrows) representing typical SIV infection were observed in all lung lobes of infected piglets. Mock-infected (MOCK) piglets did not show any gross pathologic lesions. B: Gross pathologic scores indicated that administration of $\mathrm{SCH} 527123$ plus oseltamivir significantly reduced these pathologic lesions compared with either drug administered alone. C: Rectal temperatures of piglets in mock-infected, infected (INF or I), and treatment groups. D: Body weights of animals in mock-infected, infected, and treatment groups. One-way analysis of variance, with Tukey multiple comparison tests, was performed. Results are expressed as means \pm SEM (B-D). $n=4$ piglets in MOCK, INF, I $+0 \mathrm{SL}$, and I + OSL $+\mathrm{SCH}$ groups $(\mathbf{A}-\mathbf{D}) ; n=3$ piglets in SCH treatment group $(\mathbf{A}-\mathbf{D}) .{ }^{*} P<0.05$; ${ }^{\dagger} P<0.05$ versus INF. CON, control.

activity, with prominent pathologic lesions of necrotizing bronchiolitis, disrupted basement membrane, and interstitial pneumonia. This was followed by the exudative phase between 6 and 8 dpi. As the virus spreads deeper into the lungs, the endothelium becomes a potential target for virus replication, thus destroying capillary bed disruption and causing vascular leakage and parenchymal hemorrhage. Declining neutrophil activity, NETosis, extensive presence of red blood cells, and edematous lung lesions were observed at $8 \mathrm{dpi}$, with prominent formation of hyaline membrane in the lungs between 6 and $8 \mathrm{dpi}$, indicating impaired gas exchange and respiratory failure. On the basis of these time-dependent changes of inflammatory and pathologic lesions, the efficacy of SCH527123 was assessed between 2 and $5 \mathrm{dpi}$, and massive neutrophil activation and NETs was observed between 3 and 6 dpi. Because uninfected mice showed extremely low numbers of neutrophils in the lungs, a prophylactic treatment arm was not included in this study.
Inclusion of SCH527123 significantly improved survival compared with oseltamivir treatment alone. Animals given combination drug treatment showed significant reduction in inflammation and alveolar injury, suggesting that aberrant neutrophil activity and NETs exacerbate immunopathology in severe influenza. Thus, suppressing neutrophil-mediated lung injury confers significant synergistic effects to antiviral treatment. Although these preclinical studies provide clues on the timing and dosage of SCH527123 therapy, the methods for evaluating dynamics of neutrophils and NETs proposed herein cannot be adopted in a clinical setting for patient treatment. The relatively invasive method of bronchoscopy to collect BAL may not be feasible in critically ill influenza patients. However, our data reveal correlation between pulmonary neutrophil influx and NETs with plasma CXCR2 ligands (mouse keratinocyte chemoattractant and macrophage inflammatory protein), suggesting that elevated levels of circulating CXCR2 ligands may serve as suitable biomarkers to identify inflammatory status 
A
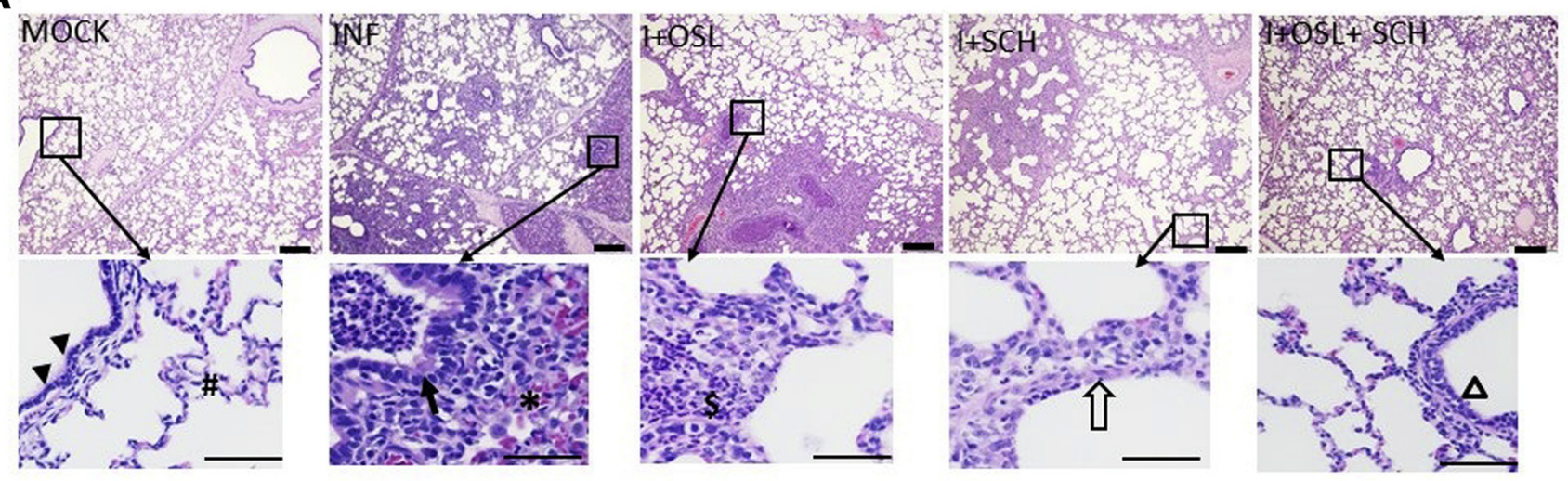

B

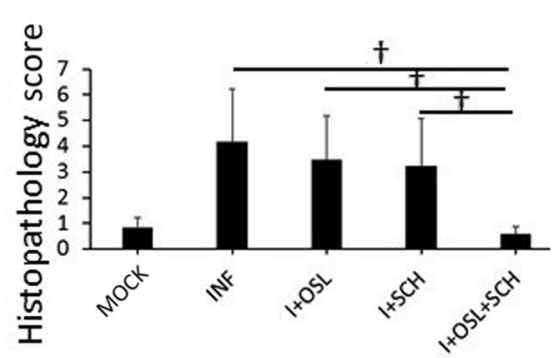

E
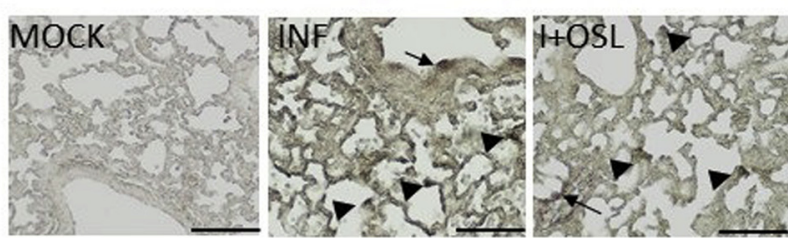

C

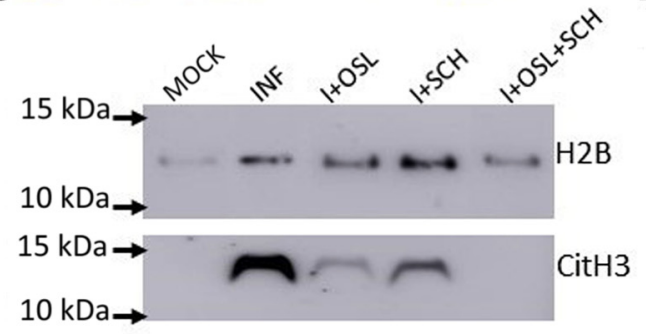

D

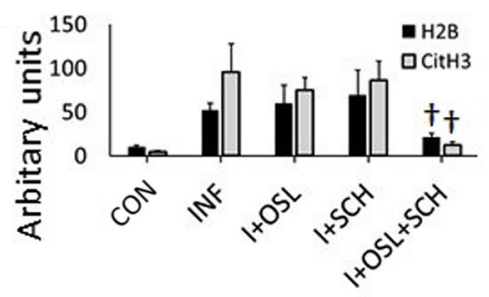

F

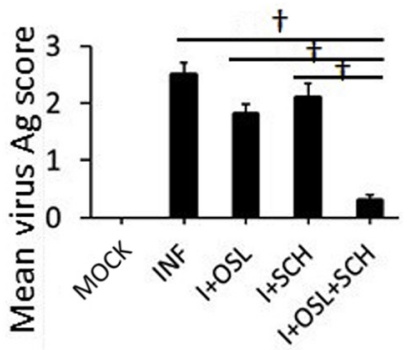

Figure 6 Treatment with SCH527123 (SCH) plus oseltamivir (OSL) reduces lung pathology, restores normal lung architecture, and inhibits viral spread in swine influenza virus (A/swine/Iowa/15/30)-infected piglets. Mock-infected (MOCK), infected (INF or I), and treated piglets were euthanized at 5 days post infection. A: Paraffin-embedded lung tissues from left lung lobes, including cranial, middle, and caudal lobes (1-cm slices from each lobe), were prepared as sections ( $6 \mu \mathrm{m}$ thick) and stained with hematoxylin and eosin, and lung injury was assessed in a blinded manner (T.A.S., N.T.). Mock-infected piglets display normal airway (arrowheads) and alveolar architecture (hash mark). Infected piglets exhibit extensive neutrophilic influx, and bronchioles appeared filled with inflammatory cellular infiltrates with necrotic epithelium (arrow), and alveolitis with extensive hemorrhage (asterisk) in damaged areas of the lungs. Piglets treated with oseltamivir alone display neutrophilic cuffing in airways (dollar sign) and alveolitis comparable with infected piglets. SCH528123 treatment alone exhibits relatively less inflammation, but airway epithelial necrosis (open arrow) is observed, which is likely due to virus-inflicted cytopathic effect. Administration of SCH527123 plus oseltamivir significantly restores normal lung architecture with intact airways (open arrowhead) and normal alveolar architecture comparable with mock-infected piglets. B: Semiquantitative analysis of histologic scores of lung sections from mock-infected, influenza-infected, and treatment groups performed in a blinded manner. C: Western blot analyses and their densitometry analyses for H2B and citrullinated histone 3 (citH3), performed on bronchoalveolar lavage samples of mock-infected, influenza-infected, and treatment groups of piglets. D: Densitometric analysis of H2B and citH3. E: Viral antigen in the lungs was detected using anti-swine influenza matrix protein 1 antibody by immunohistochemistry. Viral antigen was detected in the bronchiolar epithelial cells (arrows) and alveolar epithelial cells (arrowheads). F: Fuji-ImageJ analysis was performed to determine the mean antigen (Ag) scores in infected and treatment groups. Results are expressed as means \pm SEM (B, D, and $\mathbf{F}) . n=4$ piglets per group (MOCK, INF, I + OSL, and I $+0 S L+$ $\mathrm{SCH} ; \mathbf{A}-\mathbf{D}) ; n=3$ piglets in the I $+\mathrm{SCH}$ group $(\mathbf{A}-\mathbf{D}) .{ }^{\dagger} P<0.05$ (t-test). Scale bars $=40 \mu \mathrm{m}(\mathbf{A}$ and $\mathbf{E})$. CON, control.

in infected lungs. In addition, we previously showed elevated ECH in the nasal wash samples of influenzainfected patients. ${ }^{17}$ Hence, noninvasive techniques using nasal wash and plasma samples for measuring CXCR2 ligands, MPO/DNA, and ECH levels need to be assessed for their efficacy as biomarkers of lung inflammation and injury before considering the clinical application of CXCR2 antagonist therapy.

Our studies reveal that combinations of drugs that inhibit virus-inflicted lung pathology and neutrophil-mediated acute lung injury provide significant protection against lethal influenza. However, administration of SCH527123 alone, which 

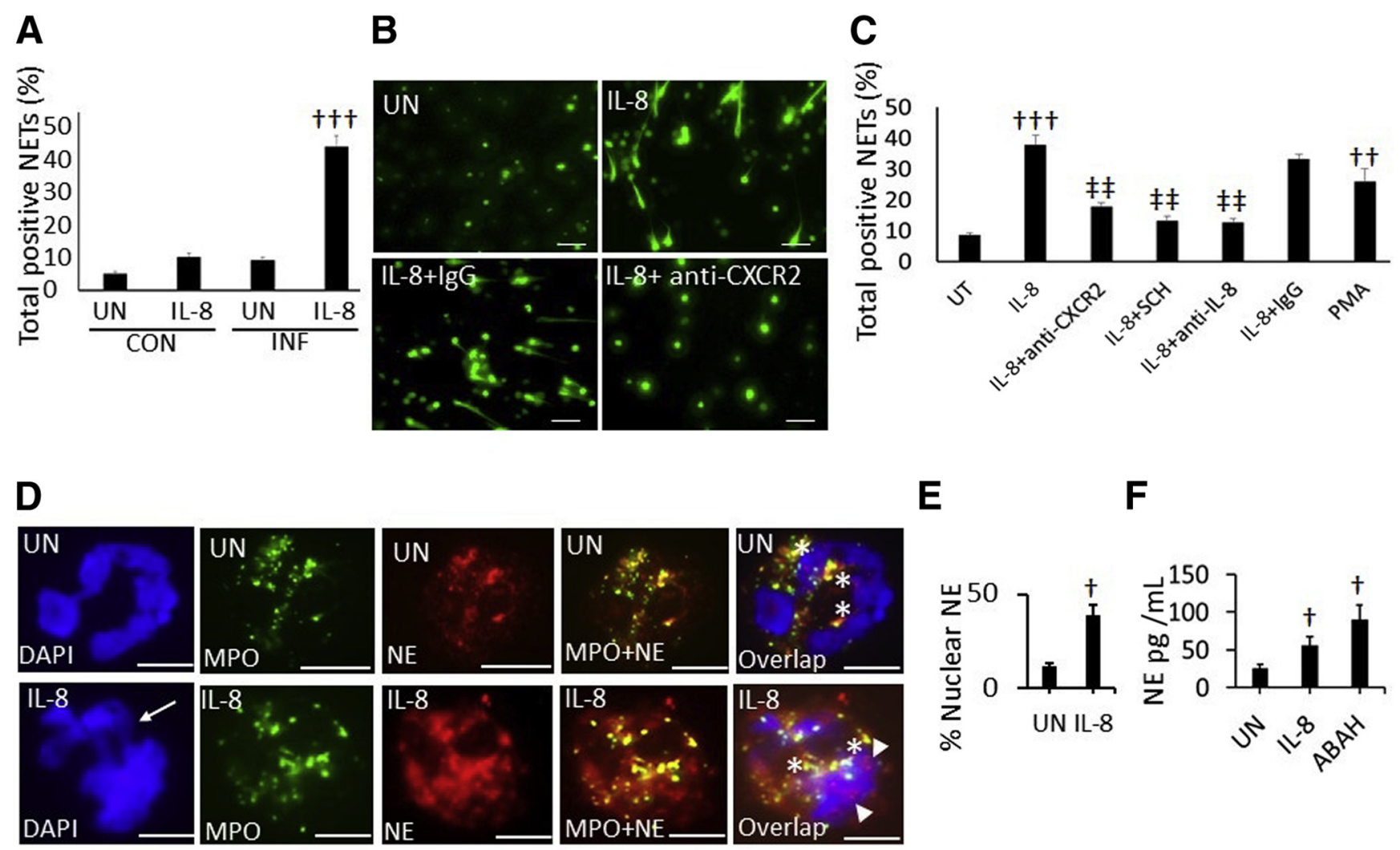

$\mathbf{E}$

$\mathbf{F}$

Figure 7 CXCR2 stimulation regulates NETosis. A: Neutrophils isolated from control (CON) and influenza-infected (INF) mice were stimulated with IL-8 $(100 \mathrm{ng} / \mathrm{mL})$ for 4 hours. IL-8 stimulation induces significant NET release in neutrophils isolated from influenza-infected mice compared with neutrophils isolated from mock-infected mice. B and C: Blocking with anti-CXCR2 antibody $(5 \mu \mathrm{g} / \mathrm{mL})$, corresponding IgG as control ( $\mathrm{g} / \mathrm{mL})$, or anti-IL-8 antibody $(5 \mu \mathrm{g} /$ $\mathrm{mL})$ or SCH527123 $(\mathrm{SCH} ; 2 \mu \mathrm{g} / \mathrm{ml})$ reveals that IL-8-induced NET formation is inhibited by anti-CXCR2 antibody, anti-IL-8 antibody, or SCH527123. D: Neutrophil elastase (NE) nuclear translocation is an essential step in NET release. To test whether induction of CXCR2 triggers NE nuclear translocation, neutrophils isolated from influenza-infected mice were stimulated with IL-8 for 3 hours, and assessed by immunofluorescence via confocal microscopy. In unstimulated neutrophils, NE staining was mainly localized in myeloperoxidase (MP0)-positive azurophilic granules (asterisks). IL-8 stimulation significantly increases NE nuclear localization (arrowheads). Disintegrated nuclei were seen during chromatin decondensation in IL-8-stimulated neutrophils (arrow). E: Quantitative assessment of NE nuclear translocation. F: IL-8 stimulation also significantly enhances NE expression, as determined by enzyme-linked immunosorbent assay, which was abrogated by MPO inhibitor, 4-aminobenzoic acid hydrazide (ABAH). Significantly increased citrullinated histone 3-positive cells are seen on IL-8 stimulation. About 20 cells were evaluated at $\times 1000$ magnification for NE nuclear translocation in unstimulated and IL-8-stimulated cells. $n=3$ experimental replicates (B-F). ${ }^{\dagger} P<0.05,{ }^{\dagger \dagger} P<0.01$, and ${ }^{\dagger \dagger \dagger} P<0.001$ versus unstimulated $\left(t\right.$-test); ${ }^{\ddagger \ddagger} P<0.01$ versus IL-8 stimulation $(t$-test). Scale bars $=20 \mu \mathrm{m}$ (B and D). PMA, phorbol 12-myristate 13-acetate; UN, unstimulated; UT, untreated.

blocks neutrophil influx and NET formation, did not alleviate morbidity and mortality in infected mice. SCH527123 treatment alone could not reduce lung damage. This may be attributed to the high viral challenge dose and widespread virus-inflicted alveolitis. However, inclusion of SCH527123 together with oseltamivir offered significant synergism by diminishing pulmonary pathology and viral loads. These results indicate the complexity of pathophysiology of influenza pneumonia, and that host-mediated factors, such as ECH and NETs, can significantly aggravate virus-inflicted cytopathic effects. Thus, preventing both host-targeted pathology and virus replication is necessary to alleviate influenza-associated lung morbidity and mortality. Congruent with these data, mice lacking peptidylarginine deiminase 4 , which are deficient in producing NETs, are susceptible to influenza, similar to wildtype mice. ${ }^{53}$ Numerous studies in influenza-infected mice have also shown promising synergistic efficacy of immunomodulatory drugs when administered together with antiviral agents. ${ }^{15,16,19,54,55}$

The murine model is commonly exploited to better understand immunopathology and to evaluate immunomodulatory drugs against influenza. However, the mouse is not a natural host for influenza virus. Therefore, further preclinical testing in natural animal hosts of influenza is required before application in humans. Validation of findings in models of larger animals (such as ferrets or pigs) is not routinely done. In our study, we validated the combination treatment in piglets infected with a swine influenza virus strain. Pigs are natural hosts for influenza, and they exhibit both humoral and cell-mediated immune responses by 4 to 5 weeks of age. ${ }^{56}$ Treatments with SCH527123 alone or together with oseltamivir were initiated at $2 \mathrm{dpi}$, and the efficacy of a three-dose regimen was evaluated. Combination treatment significantly ameliorated both gross and 
A

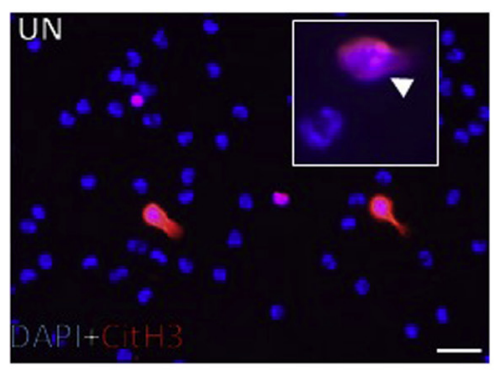

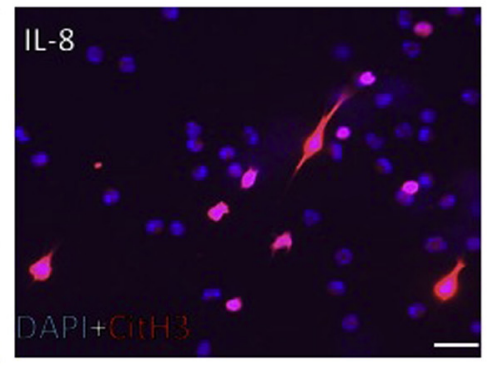

B

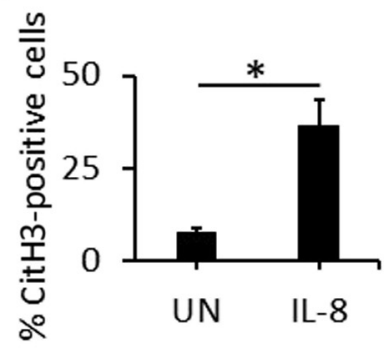

C

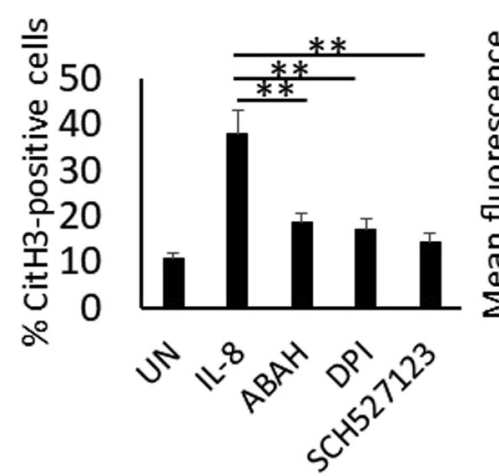

D

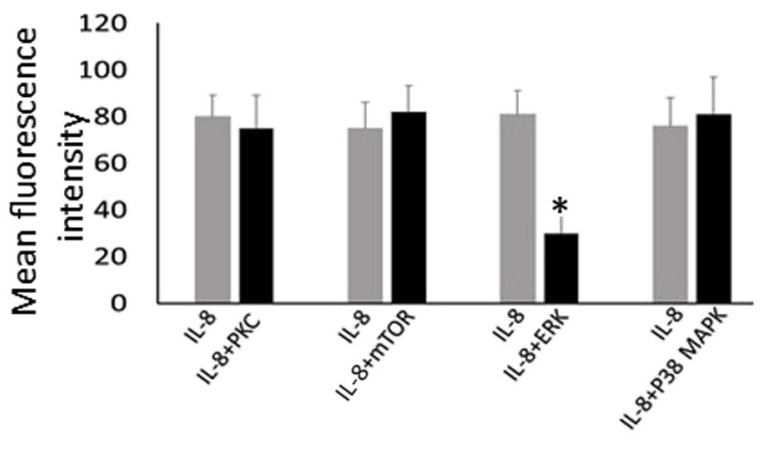

$\mathbf{E}$

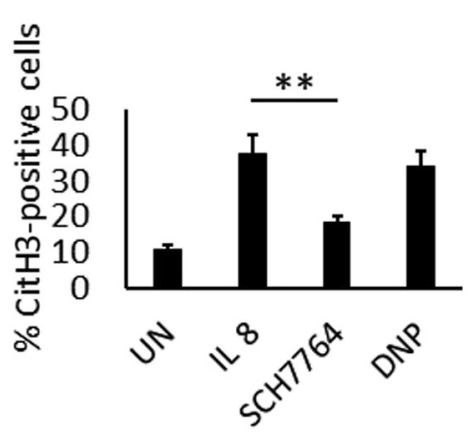

Figure 8 CXCR2-induced NETosis is mediated in an oxidation-reduction (redox) enzyme-dependent manner and is regulated by extracellular signalregulated kinase (ERK) signaling. A: Immunostaining for citrullinated histone 3 (citH3) in neutrophils stimulated with IL-8 (inset; white arrowhead indicates citH3 staining in the nucleus). Cells were counterstained with nuclear dye, DAPI. B: Quantitative assessment of citH3-positive staining in unstimulated and IL-8-stimulated neutrophils. C: Myeloperoxidase or NADPH activities were blocked using 4-aminobenzoic acid hydrazide (ABAH) and diphenyleneiodonium (DPI) and effect on IL-8-induced NETosis was evaluated. D: The downstream signaling involved in CXCR2-induced NETosis was evaluated by reactive oxygen species (ROS) induction, using various inhibitors of signaling pathways, including protein kinase C (PKC; G06983; $5 \mu \mathrm{mol} / \mathrm{L}$ ), mechanistic target of rapamycin [mTOR; 2,4-dinitrophenol (DNP); $100 \mu \mathrm{mol} / \mathrm{L}$ ], ERK (SCH772984; $10 \mu \mathrm{mol} / \mathrm{L})$, and p38 mitogen-activated protein kinase (MAPK; SB203580; 10 $\mu \mathrm{mol} / \mathrm{L})$. ROS release was measured using $2^{\prime}-7^{\prime}$-dichlorodihydrofluorecein diacetate by enzyme-linked immunosorbent assay. E: citH3 immunostaining using anti-citH3 antibody followed by counterstaining with DAPI was used to evaluate reduction in IL-8-induced NETosis in the presence of inhibitor of ERK pathway (SCH7764), or mTOR inhibitor (DNP). ${ }^{*} P<0.05,{ }^{* *} P<0.01$ (t-test). Scale bars $=20 \mu \mathrm{m}(\mathbf{A})$.

microscopic lung pathology compared with treatment with either drug alone. Mechanistically, targeting CXCR2 conferred similar protective effects of decreased alveolar epithelial injury and NETosis in both infected mice and piglets. SCH527123 alone without oseltamivir could not provide complete protection, which may be attributed to overwhelming viral infection. This indicates that simultaneous inhibition of both virus replication and host pathology is necessary to control morbidity and mortality in severe influenza. Interestingly, we found that addition of SCH527123 together with oseltamivir reduced lung viral loads in both mice and piglets. In addition, macrophages recruited into the lungs could also contribute to decreased alveolar damage and viral loads. We have previously shown that depletion of neutrophils increases macrophage-specific recruitment into the lungs, and a side from their phagocytic function, macrophages also promote alveolar repair by producing hepatocyte growth factor during influenza infection. ${ }^{57}$ Hepatocyte growth factor is a potent inducer of alveolar epithelial proliferation, and co-administration of recombinant hepatocyte growth factor and oseltamivir attenuates alveolar damage and virus replication in mice challenged with lethal influenza infection. ${ }^{22}$ The effects of CXCR2 antagonism on macrophage activity, hepatocyte growth factor release, and their involvement in alveolar repair during influenza infection warrant further investigation. Our findings provide the first evidence to support the strategy of combination therapy in a large animal influenza model, in view of the close similarities in pulmonary pathology and immune responses between swine and humans. Hence, porcine models may be useful in advancing the translational impact of drug treatment studies, and in better understanding host-directed therapy in human influenza infections.

Although several preclinical and clinical studies have tested CXCR2 antagonist treatment against influenza, ${ }^{25,26,50,51}$ the mechanisms underlying how CXCR2 antagonism exerts protection are largely unexplored. Herein, we demonstrate a novel mechanism in which CXCR2 signaling induces NETosis during influenza. Release of 
NETs is a highly controlled process involving modification of histones by citrullination and their enzymatic breakdown, which are essential for chromatin decondensation during NET release. ${ }^{44}$ Neutrophils isolated from infected mice were more susceptible to NETotic cell death on CXCR2 stimulation compared with neutrophils from healthy mice, indicating that induced CXCR2 during infection plays a critical role in influenza-induced NETs. Furthermore, CXCR2 stimulation was associated with increased NE dissociation from azurophilic granules and $\mathrm{NE}$ translocation into the nucleus, with this transmigration being blocked by an MPO inhibitor (Supplemental Figure S6). Interestingly, although IL-8 could induce NE expression, blocking MPO or NADPH had no impact on NE expression during IL-8 stimulation, indicating that oxidation-reduction enzymes do not regulate expression of $\mathrm{NE}$ but contribute to its nuclear translocation, as observed in phorbol 12-myristate 13-acetate-induced NET release. ${ }^{37}$ MPO activity is required for proteolytic activity of NE, ${ }^{39}$ and the effects of MPO on NE activity warrant further investigation. We also noted enhanced citrullination of histones on IL-8 stimulation, which could be blocked by MPO inhibitor. It would be interesting to explore if CXCR2 signaling regulates expression and activity of peptidylarginine deiminase 4 , which triggers citrullination of histones. ROS generation is essential for oxidationreduction enzyme activities during influenza-induced NET release. ${ }^{18}$ To assess the signaling pathway(s) that regulate(s) CXCR2-mediated ROS generation, protein kinase $\mathrm{C}, \mathrm{mTOR}$, ERK, and mitogen-activated protein kinase were evaluated following stimulation with IL-8. ERK inhibition significantly reduced ROS generation. Furthermore, blocking ERK also suppressed citrullination of histones, suggesting that CXCR2 stimulation requires ERK signaling activation for ROS generation during NET release. Elevated plasma levels of CXCR2 ligand, IL-8, are prominently detected in influenza-infected mice, pigs, humans, and other species, ${ }^{58,59}$ thus suggesting that plasma CXCR2 ligands may serve as early biomarkers for inflammatory response in severe influenza. Furthermore, we found that circulating neutrophils from influenza-infected mice exhibit high sensitivity for IL-8-induced NETosis. It would be informative to examine whether characterizing blood neutrophils for their sensitivity to IL-8-mediated NET formation could serve as an early marker for NETopathic injury during influenza pneumonia.

In conclusion, our findings show that combination therapy with SCH527123 plus oseltamivir significantly improved survival in lethal influenza-challenged mice, and ameliorated pulmonary pathology in swine influenzainfected piglets. Targeting CXCR2 suppressed the immunopathology via inhibition of excessive neutrophil influx, NETosis, and ECH accumulation in infected lungs. Temporal dynamics of neutrophil influx and NETs highlight the importance of capturing the inflammatory signature for CXCR2 antagonist therapy, and offer critical insights, and form the basis for the potential use of CXCR2 antagonists as therapy against severe influenza in humans.

\section{Acknowledgments}

We thank Dr. Todd Jackson, Dr. Patti Coan, Jill Murray, and Dr. Scott Carter for assistance with swine influenza virus infection studies in piglets.

\section{Author Contributions}

H.K.A., J.M.R., S.P., V.T.K.C., and N.T., conceived and designed the experiments; H.K.A., J.M.R., S.P., P.M., M.A., and N.T., acquired and analyzed the data; T.A.S., analyzed histopathology; and N.T., J.M.R., and V.T.K.C. wrote and reviewed the manuscript.

\section{Supplemental Data}

Supplemental material for this article can be found at http://doi.org/10.1016/j.ajpath.2020.12.013.

\section{References}

1. Short KR, Kroeze EJ, Fouchier RA, Kuiken T: Pathogenesis of influenza-induced acute respiratory distress syndrome. Lancet Infect Dis 2014, 14:57-69

2. Mauad T, Hajjar LA, Callegari GD, da Silva LF, Schout D, Galas FR, Alves VA, Malheiros DM, Auler JO Jr, Ferreira AF, Borsato MR, Bezerra SM, Gutierrez PS, Caldini ET, Pasqualucci CA, Dolhnikoff M, Saldiva PH: Lung pathology in fatal novel human influenza A (H1N1) infection. Am J Respir Crit Care Med 2010, 181: 72-79

3. Kumar A, Zarychanski R, Pinto R, Cook DJ, Marshall J, Lacroix J, Stelfox T, Bagshaw S, Choong K, Lamontagne F, Turgeon AF, Lapinsky S, Ahern SP, Smith O, Siddiqui F, Jouvet P, Khwaja K, McIntyre L, Menon K, Hutchison J, Hornstein D, Joffe A, Lauzier F, Singh J, Karachi T, Wiebe K, Olafson K, Ramsey C, Sharma S, Dodek P, Meade M, Hall R, Fowler RA; Canadian Critical Care Trials Group H1N1 Collaborative: Critically ill patients with 2009 influenza A (H1N1) infection in Canada. JAMA 2009, 302: 1872-1879

4. Lobo SM, Watanabe ASA, Salomão MLM, Queiroz F, Gandolfi JV, de Oliveira NE, Covello LHS, Sacillotto GH, de Godoy LG, Simões ES, Frini ICM, Da Silva Teixeira RER, Furlan NP, Dutra KR, Nogueira ML: Excess mortality is associated with influenza A (H1N1) in patients with severe acute respiratory illness. J Clin Virol 2019, 116:62-68

5. Harms PW, Schmidt LA, Smith LB, Newton DW, Pletneva MA, Walters LL, Tomlins SA, Fisher-Hubbard A, Napolitano LM, Park PK, Blaivas M, Fantone J, Myers JL, Jentzen JM: Autopsy findings in eight patients with fatal H1N1 influenza. Am J Clin Pathol 2010, 134:27-35

6. Wang H, Xiao X, Lu J, Chen Z, Li K, Liu H, Luo L, Wang M, Yang Z: Factors associated with clinical outcome in 25 patients with avian influenza A (H7N9) infection in Guangzhou, China. BMC Infect Dis 2016, 16:534S

7. Li H, Weng H, Lan C, Zhang H, Wang X, Pan J, Chen L, Huang J: Comparison of patients with avian influenza A (H7N9) and influenza A (H1N1) complicated by acute respiratory distress syndrome. Medicine (Baltimore) 2018, 97:e194 
8. Kalil AC, Thomas PG: Influenza virus-related critical illness: pathophysiology and epidemiology. Crit Care 2019, 23:258

9. Cheung CY, Leung CY, Nicholls JM: Innate immune responses to influenza A H5N1: friend or foe? Trends Immunol 2009, 12:574-584

10. Kash JC, Taubenberger JK: The role of viral, host, and secondary bacterial factors in influenza pathogenesis. Am J Pathol 2015, 185: $1528-1536$

11. Li C, Yang P, Sun Y, Li T, Wang C, Wang Z, Yan Y, Wang W, Wang C, Chen Z, Xing L, Tang C, Ju X, Guo F, Deng J, Zhao Y, Yang P, Tang J, Wang H, Zhao Z, Yin Z, Cao B, Wang X, Jiang C: IL-17 response mediates acute lung injury induced by the 2009 pandemic influenza A (H1N1) virus. Cell Res 2012, 22:528-538

12. Moscona A: Oseltamivir resistance-disabling our influenza defenses. N Engl J Med 2005, 353:2633-2636

13. Dharan NJ, Gubareva LV, Meyer JJ, Okomo-Adhiambo M, McClinton RC, Marshall SA, St George K, Epperson S, Brammer L, Klimov AI, Bresee JS, Fry AM: Infections with oseltamivir-resistant influenza A (H1N1) virus in the United States. JAMA 2009, 301: 1034-1041

14. Fukuyama S, Kawaoka Y: The pathogenesis of influenza virus infections: the contributions of virus and host factors. Curr Opin Immunol 2011, 23:481-486

15. Zheng BJ, Chan KW, Lin YP, Zhao GY, Chan C, Zhang HJ, Chen HL, Wong SS, Lau SK, Woo PC, Chan KH, Jin DY, Yuen KY: Delayed antiviral plus immunomodulator treatment still reduces mortality in mice infected by high inoculum of influenza A/H5N1 virus. Proc Natl Acad Sci U S A 2008, 105:8091-8096

16. Li R, Lim A, Phoon MC, Narasaraju TA, Ng JKW, Poh WP, Meng SK, Chow VT, Sylvie A: Attenuated Bordetella pertussis protects against highly pathogenic influenza A viruses by dampening the cytokine storm. J Virol 2010, 84:7105-7113

17. Sharma G, Sharma DC, Fen LH, Pathak M, Bethur N, Pendharkar V, Peiris M, Altmeyer R: Reduction of influenza virus-induced lung inflammation and mortality in animals treated with a phosophodisestrase-4 inhibitor and a selective serotonin reuptake inhibitor. Emerg Microbes Infect 2013, 2:e54

18. Narasaraju T, Edwin Y, Ramar perumal S, Ng HH, Poh WP, AudreyAnn L, Phoon Meng C, van Rooijen N, Chow VT: Excessive neutrophils and neutrophil extracellular traps contribute to acute lung injury of influenza pneumonitis. Am J Pathol 2011, 179: $199-210$

19. Anandi NM, Narasaraju T, Prashant R, Perumalsamy R, Tan KB, Wang S, Engelward B, Chow VT: In vivo and in vitro studies on the roles of neutrophil extracellular traps during secondary pneumococcal pneumonia after primary pulmonary influenza infection. Front Immunol 2013, 4:56

20. Ashar HK, Mueller NC, Rudd JM, Snider TA, Achanta M, Prasanthi M, Pulavendran S, Thomas PG, Ramachandran A, Malayer JR, Ritchey JW, Rajasekhar R, Chow VT, Esmon CT, Narasaraju T: Role of extracellular histones in influenza virus pathogenesis. Am J Pathol 2018, 188:135-148

21. Sivasami P, Rudd JM, Ashar HK, Maram P, Thomas PG, Akhilesh R, Malayer J, Chow VT, Teluguakula N: Platelets-neutrophil interaction is critical in NETs induction and exacerbation of pulmonary pathology during influenza pneumonia. Am J Res Cell Mol Biol 2019, 61: 689-701

22. Narasaraju TA, Edwin YY, Ng HH, Audrey A, Phoon MC, Chow VT: Therapeutic effect of combining hepatocyte growth factor and oseltamivir in amelioration of influenza pneumonitis. Curr Mol Med 2014, 14:1-13

23. Audrey-Ann L, Narasaraju T, Tan KB, Wang S, Phoon MC, Chow VT: Caspase-1-deficient mice are more susceptible to influenza pneumonitis. Eur J Inflam 2014, 12:117-130

24. Tang BM, Shojaei M, Teoh S, Meyers A, Ho J, Ball TB, Keynan Y, Pisipati A, Kumar A, Eisen DP, Lai K, Gillett M, Santram R, Geffers R, Schreiber J, Mozhui K, Huang S, Parnell GP, Nalos M, Holubova M, Chew T, Booth D, Kumar A, McLean A, Schughart K: Neutrophils- related host factors associated with severe disease and fatality in patients with influenza infection. Nat Commun 2019, 10:3422

25. Zhu L, Liu L, Zhang Y, Pu L, Liu J, Li X, Chen Z, Hao Y, Wang B, Han J, Li G, Liang S, Xiong H, Zhena, Li A, Xu J, Zeng H: High level of neutrophil extracellular traps correlates with poor prognosis of severe influenza A infection. J Infect Dis 2018, 217: 428-437

26. Rudd JM, Pulavendran S, Ashar HK, Ritchey JW, Snider TA, Malayer JR, Marie M, Chow VT, Narasaraju T: Neutrophils induce a novel chemokine receptors repertoire during influenza pneumonia. Front Cell Infect Microbiol 2019, 9:108

27. Roberts G, Chen S, Yates P, Madan A, Walker J, Washburn ML, Peat AJ, Kerwin EW, Roy-Ghanta S: Randomized, double-blind, placebo-controlled study of the safety, tolerability, and clinical effect of danirixin in adults with acute, uncomplicated influenza. Open Forum Infect Dis 2019, 6:ofz072

28. Madan A, Chen S, Yates P, Washburn ML, Roberts G, Peat AJ, Tao Y, Parry MF, Barnum O, McClain MT, Roy-Ghanta S: Efficacy and safety of danirixin (GSK1325756) co-administered with standard-of-care antiviral (oseltamivir): a phase $2 \mathrm{~b}$, global, randomized study of adults hospitalized with influenza. Open Forum Infect Dis 2019, 6:ofz163

29. Holz O, Khalilieh S, Ludwig-Sengpiel A, Watz H, Stryszak P, Soni P, Tsai M, Sadeh J, Magnussen H: SCH527123, a novel CXCR2 antagonist, inhibits ozone-induced neutrophilia in healthy subjects. Eur Respir J 2010, 35:564-570

30. Todd CM, Salter BM, Murphy DM, Watson RM, Howie KJ, Milot J, Sadeh J, Boulet LP, O'Byrne PM, Gauvreau GM: The effects of a CXCR1/CXCR2 antagonist on neutrophil migration in mild atopic asthmatic subjects. Pulm Pharmacol Ther 2016, 41:34-39

31. O'Byrne PM, Metev H, Puu M, Richter K, Keen C, Uddin M, Larsson B, Cullberg M, Nair P: Efficacy and safety of a CXCR2 antagonist, AZD5069, in patients with uncontrolled persistent asthma: a randomised, double-blind, placebo-controlled trial. Lancet Respir Med 2016, 4:797-806

32. Brinkmann V, Reichard U, Goosmann C, Fauler B, Uhlemann Y, Weiss DS, Weinrauch Y, Zychlinsky A: Neutrophil extracellular traps kill bacteria. Science 2004, 303:1532-1535

33. Sivanandham R, Brocca-Cofano E, Krampe N, Falwell E, Venkatraman S, Ribeiro RM, Apetrei C, Pandrea I: Neutrophil extracellular trap production contributes to pathogenesis in SIVinfected nonhuman primates. J Clin Invest 2018, 128:5178-5183

34. Wong SL, Demers M, Martinod K, Gallant M, Wang Y, Goldfine AB, Wagner DD: Diabetes primes neutrophils to undergo NETosis, which impairs wound healing. Nat Med 2015, 21:815-819

35. Muraro SP, De Souza GF, Gallo SW, Da Silva BK, De Oliveira SD, Vinolo M, Porto BN: Respiratory syncytial virus induces the classical ROS-dependent NETosis through PAD-4 and necroptosis pathways activation. Sci Rep 2018, 8:14166

36. Colón DF, Wanderley CW, Franchin M, Silva CM, Hiroki $\mathrm{CH}$, Castanheira F, Cunha FQ: Neutrophil extracellular traps (NETs) exacerbate severity of infant sepsis. Crit Care 2019, 23:113

37. Papayannopoulos V, Metzler KD, Hakkim A, Zychlinsky A: Neutrophil elastase and myeloperoxidase regulate the formation of neutrophil extracellular traps. J Cell Biol 2010, 191:677-691

38. Metzler Kathleen D, Goosmann C, Lubojemska A, Zychlinsky A, Papayannopoulos V: A myeloperoxidase-containing complex regulates neutrophil elastase release and actin dynamics during NETosis. Cell Rep 2014, 8:883-896

39. Rowe T, Abernathy RA, Hu-Primmer J, Thompson WW, Lu X, Lim W, Fukda K, Cox NJ, Katz JM: Detection of antibody to avian influenza A (H5N1) virus in human serum by using a combination of serologic assays. J Clin Microbiol 1999, 37:937-943

40. Nguyen JT, Smee DF, Barnard DL, Julander JG, Gross M, de Jong MD, Went GT: Efficacy of combined therapy with amantadine, oseltamivir, and ribavirin in vivo against susceptible and amantadineresistant influenza A viruses. PLoS One 2012, 7:e31006 
41. Ma J, Shen H, Liu Q, Bawa B, Qi W, Duff M, Lang Y, Lee J, Yu H, Bai J, Tong G, Hesse RA, Richt JA, Ma W: Pathogenicity and transmissibility of novel reassortant $\mathrm{H} 3 \mathrm{~N} 2$ influenza viruses with 2009 pandemic H1N1 genes in pigs. J Virol 2015, 89:2831-2841

42. Lyoo KS, Kim JK, Jung K, Kang BK, Song D: Comparative pathology of pigs infected with Korean H1N1, H1N2, or H3N2 swine influenza A viruses. Virol J 2014, 11:170

43. Reed LJ, Muench H: A simple method for estimating fifty percent endpoints. Am J Hyg 1938, 27:493-497

44. Metzler KD, Fuchs TA, Nauseef WM, Reumaux D, Roesler J, Schulze I, Wahn V, Papayannopoulos V, Zychlinsky A: Myeloperoxidase is required for neutrophil extracellular trap formation: implications for innate immunity. Blood 2011, 117:953-959

45. Khatri M, Dwivedi V, Krakowka S, Manickam C, Ali A, Wang L, Qin Z, Renukaradhya GJ, Lee CW: Swine influenza H1N1 virus induces acute inflammatory immune responses in pig lungs: a potential animal model for human H1N1 influenza virus. J Virol 2010, $84: 11210-11218$

46. Trebbien R, Larsen LE, Viuff BM: Distribution of sialic acid receptors and influenza A virus of avian and swine origin in experimentally infected pigs. Virol J 2011, 8:434

47. Rajao DS, Vincent AL: Swine as a model for influenza A virus infection and immunity. ILAR J 2015, 56:44-52

48. Yuen KY, Chan PK, Peiris M, Tsang DN, Que TL, Shortridge KF, Cheung PT, To WK, Ho ET, Sung R, Cheng AF: Clinical features and rapid viral diagnosis of human disease associated with avian influenza A H5N1 virus. Lancet 1998, 351:467-471

49. Ng HH, Narasaraju T, Sim MK, Chow VT: Doxycyline treatment attenuates acute lung injury in mice infected with highly virulent influenza H3N2 virus: involvement of matrix metalloproteases. Exp Mol Pathol 2012, 92:287-295

50. Peiris JS, Yu WC, Leung CW, Cheung CY, Ng WF, Nicholls JM, Ng TK, Chan KH, Lai ST, Lim WL, Yuen KY, Guan Y: Re-emergence of fatal human influenza A subtype H5N1 disease. Lancet 2004, 363:617-669

51. Tavares LP, Garcia CC, Machado MG, Queiroz-Junior CM, Barthelemy A, Trottein F, Siqueira MM, Brandolini L, Allegretti M,
Machado AM, de Sousa LP, Teixeira MM: CXCR1/2 antagonism is protective during influenza and post-influenza pneumococcal infection. Front Immunol 2017, 8:1799

52. Washburn ML, Crosby R, Remlinger K, Wang F, Creech D: Therapeutically attenuating neutrophil recruitment with a CXCR2 antagonist in combination with oseltamivir ameliorates influenza-induced lung injury and disease. Open Forum Infect Dis 2019, 6:ofz106

53. Tate MD, Deng YM, Jones JE, Anderson GP, Brooks AG, Reading PC: Neutrophils ameliorate lung injury and the development of severe disease during influenza infection. J Immunol 2009, 183: $7441-7450$

54. Hemmers S, Teijaro JR, Arandjelovic S, Mowen KA: PAD4mediated neutrophil extracellular trap formation is not required for immunity against influenza infection. PLoS One 2011, 6:e22043

55. Wang CH, Chung FT, Lin SM, Huang SY, Chou CL, Lee KY, Lin TY, Kuo HP: Adjuvant treatment with a mammalian target of rapamycin inhibitor, sirolimus, and steroids improves outcomes in patients with severe H1N1 pneumonia and acute respiratory failure. Crit Care Med 2014, 42:313-321

56. Jia X, Liu B, Bao L, Lv Q, Li F, Li H, An Y, Zhang X, Cao B, Wang C: Delayed oseltamivir plus sirolimus treatment attenuates H1N1 virus-induced severe lung injury correlated with repressed NLRP3 inflammasome activation and inflammatory cell infiltration. PLoS Pathog 2018, 14:e1007428

57. Narasaraju T, Ng HH, Phoon MC, Chow VT: MCP-1 antibody treatment enhances damage and impedes repair of the alveolar epithelium in influenza. Am J Res Cell Mol Biol 2010, 42: $732-743$

58. Pomorska-Mól M, Markowska-Daniel I, Kwit K, Czyżewska E, Dors A, Rachubik J, Pejsak Z: Immune and inflammatory response in pigs during acute influenza caused by H1N1 swine influenza virus. Arch Virol 2014, 159:2605-2614

59. de Jong MD, Simmons CP, Thanh TT, Hien VM, Smith GJ, Chau TN, Hoang DM, Chau NV, Khanh TH, Dong VC, Qui PT, Cam BV, Ha do Q, Guan Y, Peiris JS, Chinh NT, Hien TT, Farrar J: Fatal outcome of human influenza A (H5N1) is associated with high viral load and hypercytokinemia. Nat Med 2006, 12:1203-1207 